\title{
A General Framework for Enhancing Sparsity of Generalized Polynomial Chaos Expansions
}

\author{
Xiu Yang*1, Xiaoliang Wan ${ }^{\dagger 2}$, Lin $\operatorname{Lin}^{\ddagger 3}$, and Huan Lei ${ }^{\S 4}$ \\ ${ }^{1}$ Advanced Computing, Mathematics and Data Division, Pacific Northwest National \\ Laboratory, Richland, WA, 99352. \\ ${ }^{2}$ Department of Mathematics and Center of Computation and Technology, Louisiana \\ State University, Baton Rouge, LA, 70803. \\ ${ }^{3}$ Department of Mathematics, University of California, Berkeley and Computational \\ Research Division, Lawrence Berkeley National Laboratory, Berkeley, CA 94720. \\ ${ }^{4}$ Advanced Computing, Mathematics and Data Division, Pacific Northwest National \\ Laboratory, Richland, WA, 99352.
}

November 28, 2018

\begin{abstract}
Compressive sensing has become a powerful addition to uncertainty quantification when only limited data are available. In this paper, we provide a general framework to enhance the sparsity of the representation of uncertainty in the form of generalized polynomial chaos expansion. We use an alternating direction method to identify new sets of random variables through iterative rotations so the new representation of the uncertainty is sparser. Consequently, we increase both the efficiency and accuracy of the compressive-sensing-based uncertainty quantification method. We demonstrate that the previously developed rotationbased methods 30, 56 to enhance the sparsity of Hermite polynomial expansion is a special case of this general framework. Moreover, we use Legendre and Chebyshev polynomial expansions to demonstrate the effectiveness of this method with applications in solving stochastic partial differential equations and high-dimensional $(\mathcal{O}(100))$ problems.

keywords: Uncertainty quantification, generalized polynomial chaos, compressive sensing, iterative rotation, alternating direction.
\end{abstract}

\section{Introduction}

Surrogate-model-based uncertainty quantification (UQ) plays an important role in constructing computational models as it helps to understand the influence of uncertainties on the quantity of interest (QoI). In this paper, we focus on parametric uncertainty, i.e., some of the parameters in the system are random variables. We assume that these random variables are defined on a probability space $(\Omega, \mathcal{F}, P)$, where $\Omega$ is the event space and $P$ is a probability measure on the $\sigma$-field $\mathcal{F}$. We consider a system depending on a $d$-dimensional random vector $\boldsymbol{\xi}(\omega)=\left(\xi_{1}(\omega), \xi_{2}(\omega), \cdots, \xi_{d}(\omega)\right)^{\top}$, where $\omega$ is an event in $\Omega$. For simplicity,

\footnotetext{
*(Corresponding author) xiu.yang@pnnl.gov

†xlwan@math.lsu.edu

${ }^{\ddagger}$ linlin@math.berkeley.edu

§huan.lei@pnnl.gov
} 
we denote $\xi_{i}(\omega)$ as $\xi_{i}$. A useful surrogate model of the $\mathrm{QoI} u(\boldsymbol{\xi})$ is the generalized polynomial chaos (gPC) expansion [20, 50:

$$
u(\boldsymbol{\xi})=\sum_{n=1}^{N} c_{n} \psi_{n}(\boldsymbol{\xi})+\varepsilon(\boldsymbol{\xi}),
$$

where $\varepsilon$ is the truncation error, $N$ is a positive integer, $c_{n}$ are coefficients, and $\psi_{n}$ are multivariate polynomials which are orthonormal with respect to the measure of $\boldsymbol{\xi}$ :

$$
\int_{\mathbb{R}^{d}} \psi_{i}(\boldsymbol{x}) \psi_{j}(\boldsymbol{x}) \rho_{\boldsymbol{\xi}}(\boldsymbol{x}) \mathrm{d} \boldsymbol{x}=\delta_{i j},
$$

where $\rho_{\boldsymbol{\xi}}(\boldsymbol{x})$ is the probability density function (PDF) of $\boldsymbol{\xi}$ and $\delta_{i j}$ is the Kronecker delta function. This approximation converges in the $L_{2}$ sense as $N$ increases if $u$ is in the Hilbert space associated with the measure of $\boldsymbol{\xi}$ (i.e., the weight of the inner product is the PDF of $\boldsymbol{\xi}$ ) [50, 7, 35, 19. Both intrusive methods (e.g., stochastic Galerkin) and non-intrusive methods (e.g., probabilistic collocation method) are developed [20, 50, 44, 49, 4 ] to compute the gPC coefficients $\boldsymbol{c}=\left(c_{1}, c_{2}, \cdots, c_{N}\right)^{\top}$. Specifically, the non-intrusive method is more suited to study complex system as it does not require modifying the computational models or simulation, codes while the intrusive method requires rewriting these models or codes.

Non-intrusive methods use the samples of input $\left\{\boldsymbol{\xi}^{q}\right\}_{q=1}^{M}$ and corresponding output of the computational model $\left\{u^{q}=u\left(\boldsymbol{\xi}^{q}\right)\right\}_{q=1}^{M}$ to compute the coefficients $\boldsymbol{c}$. Subsequently, we can write Eq. (1) as the following linear system:

$$
\Psi c=u-\varepsilon,
$$

where $\boldsymbol{u}=\left(u^{1}, u^{2}, \cdots, u^{M}\right)^{\top}$ is the vector of output samples, $\boldsymbol{c}=\left(c_{1}, c_{2}, \cdots, c_{N}\right)^{\top}$ is the vector of gPC coefficients, $\boldsymbol{\Psi}$ is an $M \times N$ matrix with $\Psi_{i j}=\psi_{j}\left(\boldsymbol{\xi}^{i}\right)$ and $\varepsilon=\left(\varepsilon^{1}, \varepsilon^{2}, \cdots, \varepsilon^{M}\right)^{\top}$ is a vector of error samples with $\varepsilon^{q}=\varepsilon\left(\boldsymbol{\xi}^{q}\right)$. In many practical problems, it is costly to obtain $u^{q}$ because of the model complexity and the limited computational resources. As such, we often must consider the scenario when $M<N$ or even $M \ll N$. Namely, the number of samples is smaller than the number of basis functions, which implies that Eq. (3) is underdetermined. The compressive sensing method is effective at solving this type of underdetermined problem when $c$ is sparse [9, 17, 8, 6] and recent studies have applied this approach to UQ problems [18, 52, 55, 30, 51, 41, 37, 29. Several useful approaches have been developed to improve the efficiency of solving Eq. (3) in UQ applications. For example, weighted/re-weighted $\ell_{1}$ minimization assigns a weight to each $c_{n}$ and solves a weighted $\ell_{1}$ minimization problem to enhance the sparsity [11, 55, 36, 39]; better sampling strategies can improve the property of $\boldsymbol{\Psi}[38,22,2$, 26]; adaptive basis selection reduces the number of unknown [25, 13, 15, 3, 23]; dimension reduction techniques also reduce the number of unknown [57, 46].

We proposed to enhance the sparsity $\boldsymbol{c}$ for an arbitrarily distributed random variable using a non-linear mapping via optimization in [58. Later, motivated by quantifying the QoIs of complex biomolecule systems, we proposed an approach to enhance the sparsity of $\boldsymbol{c}$ by unitary transform of the random vector $\boldsymbol{\xi}\left[30\right.$ ] when $\xi_{i}$ are independent and identically distribution (i.i.d.) Gaussian random variables. Subsequently, we developed an iterativerotation algorithm [56] to enhance the sparsity of $\boldsymbol{c}$ more effectively in a successive manner. In this work, we provide a general framework to enhance the sparsity of the representation of uncertainty in the form of gPC expansion, and $\boldsymbol{\xi}$ are not restricted to Gaussian random variables. We use an alternating direction method to iteratively identify a rotation matrix $\mathbf{A}$, which transforms $\boldsymbol{\xi}$ to a new set of random variables $\boldsymbol{\eta}=\mathbf{A} \boldsymbol{\xi}$, where $\boldsymbol{\eta}=\left(\eta_{1}, \eta_{2}, \cdots, \eta_{d}\right)^{\top}$, such that the "gPC expansion" of $u$ with respect to $\boldsymbol{\eta}$ is sparser. In other words,

$$
u(\boldsymbol{\xi}) \approx \sum_{n=1}^{N} c_{n} \psi_{n}(\boldsymbol{\xi})=\sum_{n=1}^{N} \tilde{c}_{n} \psi_{n}(\boldsymbol{\eta}(\boldsymbol{\xi})) \approx u(\boldsymbol{\eta}(\boldsymbol{\xi})),
$$

and we intend to make $\tilde{\boldsymbol{c}}=\left(\tilde{c}_{1}, \tilde{c}_{2}, \cdots, \tilde{c}_{N}\right)^{\top}$ sparser than $\boldsymbol{c}$. By increasing the sparsity, we can improve both the efficiency and accuracy of the compressive-sensing-based UQ method 
because we can use fewer samples of $u^{q}$ to obtain a more accurate representation of $u$. Particularly, making $\tilde{\boldsymbol{c}}$ sparser than $\boldsymbol{c}$ has the potential to yield

$$
\left\|u(\boldsymbol{\xi})-\sum_{n=1}^{N} \hat{\tilde{c}}_{n} \psi_{n}(\mathbf{A} \boldsymbol{\xi})\right\|_{2} \leq\left\|u(\boldsymbol{\xi})-\sum_{n=1}^{N} \hat{c}_{n} \psi_{n}(\boldsymbol{\xi})\right\|_{2},
$$

when $\hat{\boldsymbol{c}}$ and $\hat{\tilde{\boldsymbol{c}}}$ are approximations of $\boldsymbol{c}$ and $\tilde{\boldsymbol{c}}$ by compressive sensing, respectively. This is because the compressive sensing method computes sparser coefficients more accurately with a given samples size (see Eq. 10 for the error estimate). Of note, $\psi_{n}$ may not be orthonormal with respect to the measure of $\boldsymbol{\eta}$. Thus, the new representation of $u$ with respect to $\boldsymbol{\eta}, \sum_{n=1}^{N} \tilde{c}_{n} \psi_{n}(\boldsymbol{\eta})$, is not necessarily a standard gPC expansion. Instead, it is a polynomial-based surrogate model for $u$. We use Legendre and Chebyshev polynomial expansions to demonstrate the effectiveness of our proposed method.

\section{Brief review of the compressive-sensing-based gPC method}

\subsection{Generalized polynomial chaos expansions}

In this paper, we study systems relying on $d$-dimensional i.i.d. random variables $\boldsymbol{\xi}$. Hence, the gPC basis functions are constructed by tensor products of univariate orthonormal polynomials. For a multi-index $\boldsymbol{\alpha}=\left(\alpha_{1}, \alpha_{2}, \cdots, \alpha_{d}\right), \alpha_{i} \in \mathbb{N} \cup\{0\}$, we set

$$
\psi_{\boldsymbol{\alpha}}(\boldsymbol{\xi})=\psi_{\alpha_{1}}\left(\xi_{1}\right) \psi_{\alpha_{2}}\left(\xi_{2}\right) \cdots \psi_{\alpha_{d}}\left(\xi_{d}\right) .
$$

For two different multi-indices $\boldsymbol{\alpha}_{i}=\left(\left(\alpha_{i}\right)_{1}, \cdots,\left(\alpha_{i}\right)_{d}\right)$ and $\boldsymbol{\alpha}_{j}=\left(\left(\alpha_{j}\right)_{1}, \cdots,\left(\alpha_{j}\right)_{d}\right)$, we have the property

$$
\int_{\mathbb{R}^{d}} \psi_{\boldsymbol{\alpha}_{i}}(\boldsymbol{x}) \psi_{\boldsymbol{\alpha}_{j}}(\boldsymbol{x}) \rho_{\boldsymbol{\xi}}(\boldsymbol{x}) \mathrm{d} \boldsymbol{x}=\delta_{\boldsymbol{\alpha}_{i} \boldsymbol{\alpha}_{j}}=\delta_{\left(\alpha_{i}\right)_{1}\left(\alpha_{j}\right)_{1}} \delta_{\left(\alpha_{i}\right)_{2}\left(\alpha_{j}\right)_{2}} \cdots \delta_{\left(\alpha_{i}\right)_{d}\left(\alpha_{j}\right)_{d}},
$$

where

$$
\rho_{\boldsymbol{\xi}}(\boldsymbol{x})=\rho_{\xi_{1}}\left(x_{1}\right) \rho_{\xi_{2}}\left(x_{2}\right) \cdots \rho_{\xi_{d}}\left(x_{d}\right) .
$$

For simplicity, we denote $\psi_{\boldsymbol{\alpha}_{i}}(\boldsymbol{\xi})$ as $\psi_{i}(\boldsymbol{\xi})$.

\subsection{Compressive sensing}

We first introduce the concept of sparsity as it is critical in the error estimates for solving the under-determined system Eq. (3) with the compressive sensing method. The number of non-zero entries of a vector $\boldsymbol{x}=\left(x_{1}, x_{2}, \cdots, x_{N}\right)$ is denoted as $\|\boldsymbol{x}\|_{0} \stackrel{\text { def }}{=} \#\left\{i: x_{i} \neq 0\right\}$ [16, 9, 6], and the $\ell_{1}$ norm of $\boldsymbol{x}$ is defined as $\|\boldsymbol{x}\|_{1} \stackrel{\text { def }}{=} \sum_{n=1}^{N}\left|x_{n}\right|$. Of note, $\|\cdot\|_{0}$ is named " $\ell_{0}$ norm" in [16, although it is not a norm nor a semi-norm. The vector $\boldsymbol{x}$ is called $s$-sparse if $\|\boldsymbol{x}\|_{0} \leq s$, and $\boldsymbol{x}$ is considered a sparse vector if $s \ll N$. Few practical systems have truly sparse gPC coefficients $\boldsymbol{c}$. However, in many cases, the $\boldsymbol{c}$ are compressible, i.e., only a few entries make significant contribution to its $\ell_{1}$ norm. Consequently, a vector $\boldsymbol{x}_{s}$ is defined as the best $s$-sparse approximation that can be obtained knowing the exact locations and amplitudes of the $s$-largest entries of $\boldsymbol{x}$, i.e., $\boldsymbol{x}_{s}$ is the vector $\boldsymbol{x}$ with all but the $s$-largest entries set to zero [8]. Subsequently, $\boldsymbol{x}$ is considered sparse if $\left\|\boldsymbol{x}-\boldsymbol{x}_{s}\right\|_{1}$ is small for $s \ll N$.

Under some conditions, the sparse vector $c$ in Eq. (3) can be approximated by solving the following $\ell_{1}$ minimization problem:

$$
\left(P_{1, \epsilon}\right): \arg \min _{\hat{\boldsymbol{c}}}\|\hat{\boldsymbol{c}}\|_{1}, \quad \text { subject to }\|\boldsymbol{\Psi} \hat{\boldsymbol{c}}-\boldsymbol{u}\|_{2} \leq \epsilon,
$$


where $\epsilon=\|\varepsilon\|_{2}$. The error bound for solving Eq. (3) with $\ell_{1}$ minimization requires definiting the restricted isometry property (RIP) constant [10. For each integer $s=1, \cdots, N$, the isometry constant $\delta_{s}$ of a matrix $\boldsymbol{\Phi}$ is defined as the smallest number such that

$$
\left(1-\delta_{s}\right)\|\boldsymbol{x}\|_{2}^{2} \leq\|\boldsymbol{\Phi} \boldsymbol{x}\|_{2}^{2} \leq\left(1+\delta_{s}\right)\|\boldsymbol{x}\|_{2}^{2}
$$

holds for all $s$-sparse vectors $\boldsymbol{x}$. With some restrictions, Candès et al. showed $\boldsymbol{x}$ can be stably reconstructed [8]. Assume that the matrix $\boldsymbol{\Psi}$ satisfies $\delta_{2 s}<\sqrt{2}-1$, and $\|\varepsilon\|_{2} \leq \epsilon$, then solution $\hat{\boldsymbol{c}}$ to $\left(P_{1, \epsilon}\right)$ obeys

$$
\|\boldsymbol{c}-\hat{\boldsymbol{c}}\|_{2} \leq C_{1} \epsilon+C_{2} \frac{\left\|\boldsymbol{c}-\boldsymbol{c}_{s}\right\|_{1}}{\sqrt{s}}
$$

where $C_{1}$ and $C_{2}$ are constants, $\boldsymbol{c}$ is the exact vector we aim to approximate and $\hat{\boldsymbol{c}}$ is the solution of $\left(P_{1, \epsilon}\right)$. This result implies that the upper bound of the error is related to the truncation error and the sparsity of $c$, which is indicated in the first and second terms on the right-hand side of Eq. 10 , respectively. We use $\left\|\boldsymbol{c}-\boldsymbol{c}_{s}\right\|_{1} / \sqrt{s}$ to examine the sparsity in our numerical examples.

In practice, the error term $\epsilon$ is not known a priori. Hence, in the present work, we use cross-validation to estimate it. One such algorithm is 18 summarized in Algorithm 1 . We

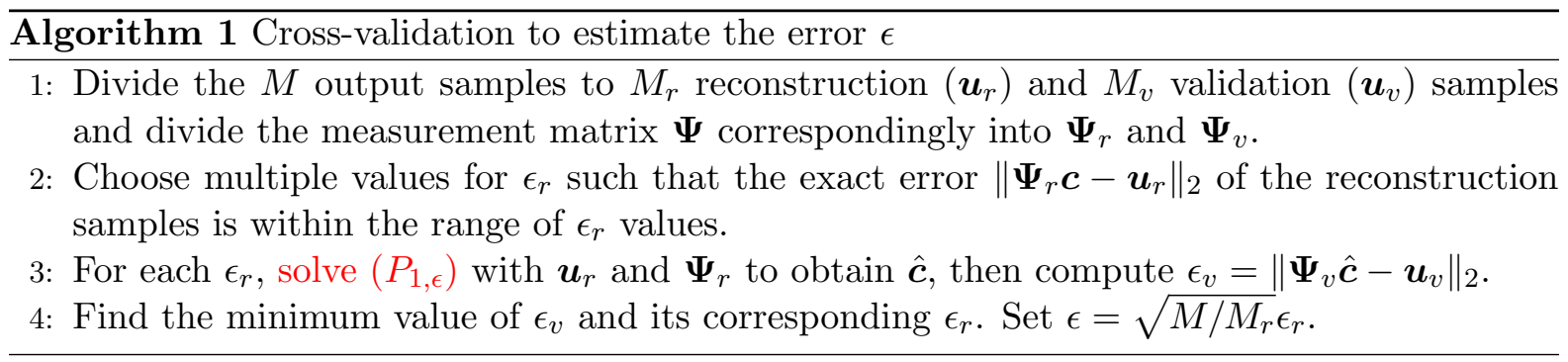

note that some techniques may be applied to avoid the cross-validation step and we refer interested readers to [1].

\subsection{Compressive-sensing-based gPC methods}

Given $M$ samples of $\boldsymbol{\xi}$, the QoI $u$ is approximated by a gPC expansion as in Eq. (1):

$$
u\left(\boldsymbol{\xi}^{q}\right)=\sum_{n=1}^{N} c_{n} \psi_{n}\left(\boldsymbol{\xi}^{q}\right)+\varepsilon\left(\boldsymbol{\xi}^{q}\right), \quad q=1,2, \cdots, M,
$$

which can be rewritten as Eq. (3). A typical approach to compressive-sensing-based-gPC is summarized in Algorithm 2 . Moreover, we use the re-weighted $\ell_{1}$ minimization approach [11] in the numerical examples to improve the accuracy of solving Eq. 8). This approach solves the following optimization problem:

$$
\left(P_{1, \epsilon}^{W}\right): \arg \min _{\hat{\boldsymbol{c}}}\|\mathbf{W} \hat{\boldsymbol{c}}\|_{1}, \text { subject to }\|\boldsymbol{\Psi} \hat{\boldsymbol{c}}-\boldsymbol{u}\|_{2} \leq \epsilon,
$$

where $\mathbf{W}$ is a diagonal matrix: $\mathbf{W}=\operatorname{diag}\left(w_{1}, w_{2}, \cdots, w_{N}\right)$. Clearly, $\left(P_{1, \epsilon}\right)$ can be considered as a special case of $\left(P_{1, \epsilon}^{W}\right)$ by setting $\mathbf{W}=\mathbf{I}$. The elements $w_{i}$ of the diagonal matrix can be estimated iteratively [11, 55]. More precisely, in the $l$-th iteration, $\left(P_{1, \epsilon}^{W}\right)$ is solved to obtain $\hat{\boldsymbol{c}}^{(l)}$. Then we set $w_{i}^{(l+1)}=1 /\left(\left|\hat{c}_{i}^{(l)}\right|+\delta\right)$ for the next iteration. The parameter $\delta>0$ is introduced to provide stability and to ensure that a zero-valued component in $\hat{\boldsymbol{c}}^{(l)}$ does not prohibit a nonzero estimate at the next step. In Candès et al. [11, the authors suggest two to three iterations of this procedure. Subsequent analytical work 34 provides an error bound for each iteration, as well as the limit of computing $\hat{\boldsymbol{c}}$ with re-weighted $\ell_{1}$ minimization. The form is similar to Eq. 10 with different constants. 


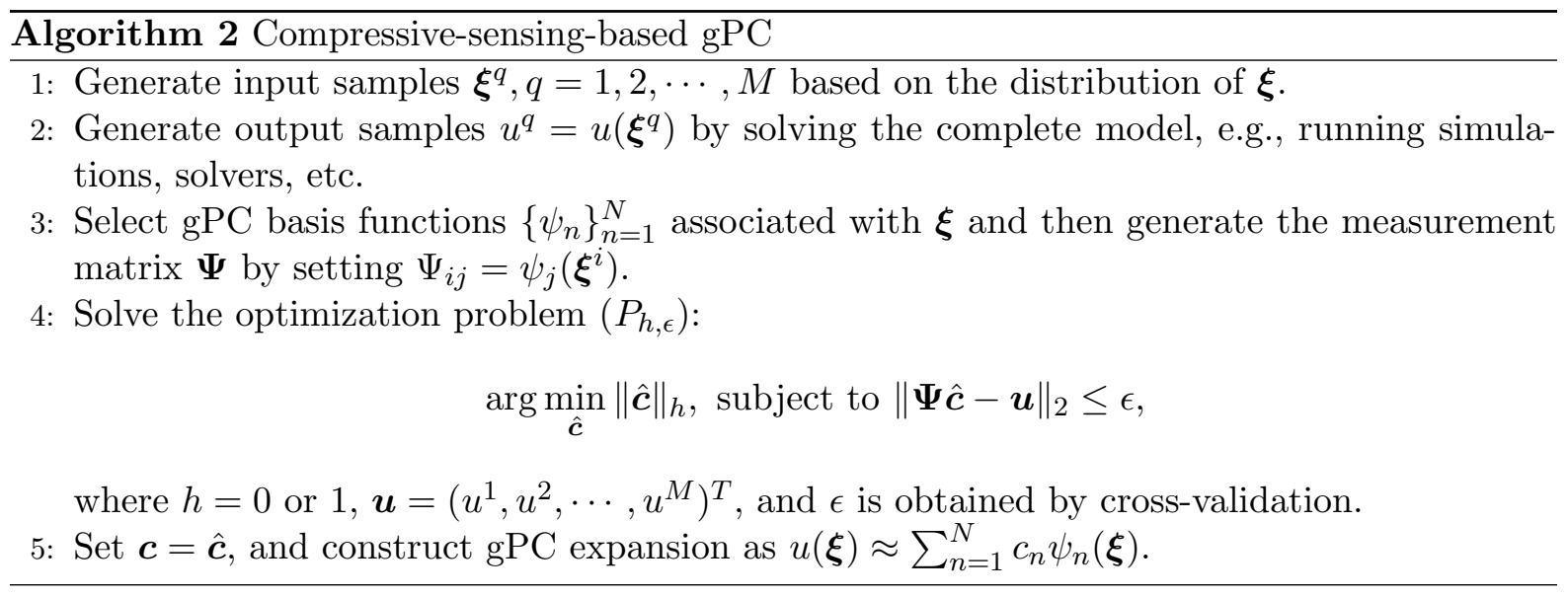

\section{Iterative rotations}

\subsection{Basic idea}

We aim to find a linear map $g: \mathbb{R}^{d} \mapsto \mathbb{R}^{d}$ such that we have a new set of random variables:

$$
\boldsymbol{\eta}=g(\boldsymbol{\xi})=\mathbf{A} \boldsymbol{\xi}, \quad \boldsymbol{\eta}=\left(\eta_{1}, \eta_{2}, \cdots, \eta_{d}\right)^{\top},
$$

where $\mathbf{A}$ is an orthogonal matrix satisfying $\mathbf{A} \mathbf{A}^{\top}=\mathbf{I}$ and the PDF of $\boldsymbol{\eta}$ is denoted as $\rho_{\boldsymbol{\eta}}$. In other words, the map from $\boldsymbol{\xi}$ to $\boldsymbol{\eta}$ can be considered as a rotation in $\mathbb{R}^{d}$. As such, the new polynomial expansion for $u$ is

$$
u(\boldsymbol{\xi}) \approx u_{g}(\boldsymbol{\xi})=\sum_{n=1}^{N} c_{n} \psi_{n}(\boldsymbol{\xi})=\sum_{n=1}^{N} \tilde{c}_{n} \psi_{n}(\mathbf{A} \boldsymbol{\xi})=\sum_{n=1}^{N} \tilde{c}_{n} \psi_{n}(\boldsymbol{\eta})=v_{g}(\boldsymbol{\eta}),
$$

namely,

$$
u(\boldsymbol{\xi})=v(\boldsymbol{\eta}(\boldsymbol{\xi}))=v(\boldsymbol{\eta}) \approx v_{g}(\boldsymbol{\eta}) .
$$

Here, $u_{g}(\boldsymbol{\xi})$ is understood as a polynomial $u_{g}(\boldsymbol{x})$ evaluated at the random variables $\boldsymbol{\xi}$ and the same for $v_{g}$. Ideally, $\tilde{\boldsymbol{c}}$ is sparser than $\boldsymbol{c}$. In the previous work [30, 56], we assume that $\boldsymbol{\xi} \sim \mathcal{N}(\mathbf{0}, \mathbf{I})$. Hence, $\boldsymbol{\eta} \sim \mathcal{N}(\mathbf{0}, \mathbf{I})$. For general cases where $\left\{\xi_{i}\right\}_{i=1}^{d}$ are not i.i.d. Gaussian, $\left\{\eta_{i}\right\}_{i=1}^{d}$ are not necessarily independent. Moreover, $\left\{\psi_{n}\right\}_{n=1}^{N}$ are not necessarily orthogonal to each other with respect to $\rho_{\boldsymbol{\eta}}$. Therefore, $v_{g}(\boldsymbol{\eta})$ may not be a standard gPC expansion of $v(\boldsymbol{\eta})$. It is a polynomial equivalent to $u_{g}(\boldsymbol{\xi})$ with potentially sparser coefficients. The idea of using a linear map is also used in sliced inverse regression (SIR) [31, active subspace [40, 12], and basis adaptation [45], while these methods compute the matrix in different manners. More importantly, in contrast to these methods, our method does not truncate the dimension, and $\mathbf{A}$ is a square matrix. We use an iterative algorithm to identify $\mathbf{A}$, and the initial guess may not be sufficiently accurate. Thus, reducing dimension before the iterations terminate may lead to less accurate results. The dimension reduction can be integrated with the iterative method proposed in this work, e.g., an algorithm for i.i.d. Gaussian random variables with dimension reduction after iterative rotations was proposed in [53. An iterative rotation method preceded with SIR-based dimension reduction was proposed in [57]. We refer interested readers to the respective literatures.

In this work, we use the gradient information of $u$ to identify the rotation matrix $\mathbf{A}$ based on the framework of active subspace [40. We define a "stiff matrix" $\mathbf{G}$ and compute its eigendecomposition as

$$
\mathbf{G} \stackrel{\text { def }}{=} \mathbb{E}\left\{\nabla u(\boldsymbol{\xi}) \cdot \nabla u(\boldsymbol{\xi})^{\top}\right\}=\mathbf{U} \boldsymbol{\Lambda} \mathbf{U}^{\top}, \quad \mathbf{U} \mathbf{U}^{\top}=\mathbf{I} .
$$

Here, $\boldsymbol{\Lambda}=\operatorname{diag}\left(\lambda_{1}, \cdots, \lambda_{d}\right), \lambda_{1} \geq \cdots \geq \lambda_{d} \geq 0, \nabla u(\boldsymbol{x})=\left(\partial u / \partial x_{1}, \cdots, \partial u / \partial x_{d}\right)^{\top}$ is a column vector, and $\nabla u(\boldsymbol{\xi})=\left.\nabla u(\boldsymbol{x})\right|_{\boldsymbol{x}=\boldsymbol{\xi}}$. We then set $\mathbf{A}=\mathbf{U}^{\top}$. The magnitude of $\lambda_{i}$ 
indicates the importance of the corresponding eigenspace. If only a few $\lambda_{i}$ are large and others are very small, we can expect to obtain a sparse $\tilde{\boldsymbol{c}}$ because only a few $\eta_{i}$ make major contributions in expansion $v_{g}(\boldsymbol{\eta})$.

In practice, it is difficult to compute the expectation in Eq. (15), which is a highdimensional integral and $u$ is unknown. Thus, we used $u_{g}$ to approximate $u$ in Eq. 115 [30, and provided an analytic form to approximate G [56] (see Section 3.3). Alternatively, in this work, we use another approach to compute the rotation matrix as in [12. We define

$$
\mathbf{W}=\frac{1}{\sqrt{M}}\left[\nabla u\left(\boldsymbol{\xi}^{1}\right), \nabla u\left(\boldsymbol{\xi}^{2}\right), \cdots, \nabla u\left(\boldsymbol{\xi}^{M}\right)\right],
$$

where $M$ is the number of available samples, and we set $M \geq d$ in this work. Thus, $\mathbf{W}$ is a $d \times M$ matrix. The singular value decomposition (SVD) or principle component analysis $(\mathrm{PCA})$ of $\mathbf{W}$ yields

$$
\mathbf{W}=\mathbf{U}_{W} \boldsymbol{\Sigma}_{W} \mathbf{V}_{W}^{\top}
$$

where $\mathbf{U}_{W}$ is a $d \times d$ orthogonal matrix and $\boldsymbol{\Sigma}_{W}$ is a $d \times M$ matrix, whose diagonal consists of singular values $\sigma_{1} \geq \cdots \geq \sigma_{d} \geq 0$. We set the rotation matrix as $\mathbf{A}=\mathbf{U}_{W}^{\top}$. As such, the rotation projects $\boldsymbol{\xi}$ to the directions of principle components of $\nabla u$. Of note, we do not use the information of the orthogonal matrix $\mathbf{V}_{W}$.

The connections between Eq. (15) and (17) are as follows: when samples $\boldsymbol{\xi}^{q}$ are generated based on the distribution of $\boldsymbol{\xi}$ and $M \rightarrow \infty$, we have $\boldsymbol{\Sigma}_{W}^{\top} \boldsymbol{\Sigma}_{W} \rightarrow \boldsymbol{\Lambda}$, i.e., $\sigma_{i}^{2} \rightarrow \lambda_{i}$. Also, the eigenspaces corresponding to $\sigma_{i}$ converge to eigenspaces corresponding to $\lambda_{i}$. In other words, the rotation identified by Eq. (17) is the same as that from Eq. 15 asymptotically. These connections are summarized in the following two lemmas.

Lemma 3.1. Assume that $\nabla u(\boldsymbol{x})$ exists for any $\boldsymbol{x} \in \mathbb{R}^{d}$, and $\nabla u$ is square-integrable with respect to the measure of $\boldsymbol{\xi}$. If the matrix $\mathbf{W}$ in Eq. (16) is constructed with the samples $\boldsymbol{\xi}^{q}$ that are generated based on the distribution of $\boldsymbol{\xi}$, then $\left\|\boldsymbol{\Sigma}_{W} \boldsymbol{\Sigma}_{W}^{\top}-\boldsymbol{\Lambda}\right\|_{F} \rightarrow 0$ as $M \rightarrow \infty$, where $\|\cdot\|_{F}$ is the Frobenius norm.

Proof. Because $\boldsymbol{\xi}^{q}$ are generated based on the distribution of $\boldsymbol{\xi},\left\|\mathbf{W} \mathbf{W}^{\top}-\mathbf{G}\right\|_{F} \rightarrow 0$ as $M \rightarrow \infty$. This is straightforward because it uses the Monte Carlo method to approximate integrals. Then, because of the following eigendecomposition

$$
\mathbf{W} \mathbf{W}^{\top}=\mathbf{U}_{W}\left(\boldsymbol{\Sigma}_{W} \boldsymbol{\Sigma}_{W}^{\top}\right) \mathbf{U}_{W}^{\top},
$$

according to Hoffman and Wielandt [24, we have

$$
\left\|\boldsymbol{\Sigma}_{W} \boldsymbol{\Sigma}_{W}^{\top}-\boldsymbol{\Lambda}\right\|_{F}=\sqrt{\sum_{i=1}^{d}\left(\sigma_{i}^{2}-\lambda_{i}\right)^{2}} \leq\left\|\mathbf{W} \mathbf{W}^{\top}-\mathbf{G}\right\|_{F} \rightarrow 0 .
$$

To demonstrate the convergence of eigenspaces, we consider the case when $\mathbf{G}$ has at least two distinct eigenvalues. Otherwise, all eigenvector directions are equally important, and the rotation can't enhance the sparsity. Assume without losing generality that $\lambda_{1}=\lambda_{2}=$ $\cdots=\lambda_{k}>\lambda_{k+1}$, where $0<k<d$. We rewrite Eq. 15 as

$$
\mathbf{G}=\mathbf{U} \boldsymbol{\Lambda} \mathbf{U}^{\top}=\left(\mathbf{U}_{1}, \mathbf{U}_{2}\right)\left(\begin{array}{cc}
\boldsymbol{\Lambda}_{1} & 0 \\
0 & \boldsymbol{\Lambda}_{2}
\end{array}\right)\left(\begin{array}{l}
\mathbf{U}_{1}^{\top} \\
\mathbf{U}_{2}^{\top}
\end{array}\right),
$$

where $\boldsymbol{\Lambda}_{1}=\operatorname{diag}\left(\lambda_{1}, \lambda_{2}, \cdots, \lambda_{k}\right)$, and $\mathbf{U}_{1}$ consists of basis of eigenspace corresponding to $\lambda_{1}$, i.e., the first $k$ columns of $\mathbf{U}$. Accordingly, Eq. (18) can be written as

$$
\mathbf{W} \mathbf{W}^{\top}=\mathbf{U}_{W}\left(\boldsymbol{\Sigma}_{W} \boldsymbol{\Sigma}_{W}^{\top}\right) \mathbf{U}_{W}^{\top}=\left(\widetilde{\mathbf{U}}_{1}, \widetilde{\mathbf{U}}_{2}\right)\left(\begin{array}{cc}
\widetilde{\boldsymbol{\Lambda}}_{1} & 0 \\
0 & \widetilde{\boldsymbol{\Lambda}}_{2}
\end{array}\right)\left(\begin{array}{c}
\widetilde{\mathbf{U}}_{1}^{\top} \\
\widetilde{\mathbf{U}}_{2}^{\top}
\end{array}\right),
$$


where $\widetilde{\boldsymbol{\Lambda}}_{1}=\operatorname{diag}\left(\sigma_{1}^{2}, \sigma_{2}^{2}, \cdots, \sigma_{k}^{2}\right)$, and $\widetilde{\mathbf{U}}_{1}$ consists of the first $k$ columns of $\mathbf{U}_{W}$. We denote the subspace spanned by columns of $\mathbf{U}_{1}$ as $\mathbb{H}$, and the subspace spanned by columns of $\widetilde{\mathbf{U}}_{1}$ as $\widetilde{\mathbb{H}}$. We demonstrate that the principle angles (or canonical angles) 43 between $\mathbb{H}$ and $\widetilde{\mathbb{H}}$ convergence to 0 as $M \rightarrow \infty$, which indicates that $\widetilde{\mathbb{H}}$ converges to $\mathbb{H}$.

Lemma 3.2. Let $\mathbf{U}_{1}$ and $\widetilde{\mathbf{U}}_{1}$ be defined in Eq. 19) and Eq. 20. The angle matrix between $\mathbf{U}_{1}$ and $\widetilde{\mathbf{U}}_{1}$ is defined as

$$
\boldsymbol{\Theta}\left(\mathbf{U}_{1}, \widetilde{\mathbf{U}}_{1}\right) \stackrel{\text { def }}{=} \arccos \left(\mathbf{U}_{1}^{\top} \widetilde{\mathbf{U}}_{1} \widetilde{\mathbf{U}}_{1}^{\top} \mathbf{U}_{1}\right)^{-1 / 2} .
$$

We have $\left\|\sin \boldsymbol{\Theta}\left(\mathbf{U}_{1}, \widetilde{\mathbf{U}}_{1}\right)\right\|_{F} \rightarrow 0$ as $M \rightarrow \infty$.

Proof. Let $\beta=\min _{1 \leq j \leq d-k}\left|\lambda_{k}-\lambda_{k+j}\right| / 2>0$. According to Lemma 3.1, there exists an integer $M_{0}>0$, such that for any $M>M_{0}, \max _{1 \leq i \leq d}\left|\sigma_{i}^{2}-\lambda_{i}\right|<\beta$. Therefore, $\min _{1 \leq i \leq k, 1 \leq j \leq d-k} \mid \lambda_{i}-$ $\sigma_{k+j}^{2} \mid>\beta$ for any $M>M_{0}$. According to [14], we have

$$
\left\|\sin \boldsymbol{\Theta}\left(\mathbf{U}_{1}, \widetilde{\mathbf{U}}_{1}\right)\right\|_{F} \leq \frac{\left\|\left(\mathbf{G}-\mathbf{W} \mathbf{W}^{\top}\right) \mathbf{U}_{1}\right\|_{F}}{\beta} \leq \frac{\left\|\mathbf{G}-\mathbf{W} \mathbf{W}^{\top}\right\|_{F}\left\|\mathbf{U}_{1}\right\|_{F}}{\beta} \rightarrow 0 .
$$

The principle angles are singular values of $\boldsymbol{\Theta}\left(\mathbf{U}_{1}, \widetilde{\mathbf{U}}_{1}\right)$, and Lemma 3.2 indicates that they converge to 0 . Thus, $\widetilde{\mathbb{H}}$ converges to $\mathbb{H}$. Similarly, the eigenspace associated with other singular values of $\mathbf{W}$ converges to eigenspace associated with corresponding eigenvalues of G.

Because $u$ is not known and we assume that samples of $\nabla u$ are not available, we replace $u$ with $u_{g}$ in Eq. 16] for approximation:

$$
\mathbf{W} \approx \mathbf{W}_{g}=\frac{1}{\sqrt{M}}\left[\nabla u_{g}\left(\boldsymbol{\xi}^{1}\right), \nabla u_{g}\left(\boldsymbol{\xi}^{2}\right), \cdots, \nabla u_{g}\left(\boldsymbol{\xi}^{M}\right)\right]
$$

and the rotation matrix is constructed based on the SVD of $\mathbf{W}_{g}$ :

$$
\mathbf{W}_{g}=\mathbf{U}_{W_{g}} \boldsymbol{\Sigma}_{W_{g}} \mathbf{V}_{W_{g}}^{\top}, \quad \mathbf{A}=\mathbf{U}_{W_{g}}^{\top} .
$$

Here, $u_{g}$ can be computed with different methods, and we use standard $\ell_{1}$ (or re-weighted $\ell_{1}$ ) minimization to compute it. On obtaining $\mathbf{A}$, we define $\boldsymbol{\eta}=\mathbf{A} \boldsymbol{\xi}$ and compute the corresponding input samples as $\boldsymbol{\eta}^{q}=\mathbf{A} \boldsymbol{\xi}^{q}$. After, we construct a new measurement matrix $\boldsymbol{\Psi}(\boldsymbol{\eta})$ as $(\boldsymbol{\Psi}(\boldsymbol{\eta}))_{i j}=\psi_{j}\left(\boldsymbol{\eta}^{i}\right)$. Next, we solve the $\ell_{1}$ minimization problem $\left(P_{1, \varepsilon}\right)$ to obtain $\tilde{\boldsymbol{c}}$. If a few singular values of $\mathbf{W}$ are much larger than others, we can expect that the representation of $u$ with respect to $\boldsymbol{\eta}$ mainly replies on the eigenspace associated with these singular values, i.e., $\eta_{i}$ corresponding to large $\sigma_{i}$. Therefore, we can obtain a sparser representation. On the other hand, if the differences between $\sigma_{i}$ are small, the rotation will not enhance the sparsity, and this method may not result in a more accurate representation of uncertainty.

\subsection{Iterative method}

We generalize the ideas in Section 3.1 and formulate it as the following modified $\ell_{1}$ minimization problem:

$$
\left(P_{1, \varepsilon}^{R}\right) \quad \underset{\tilde{\boldsymbol{c}}, \mathbf{A}}{\arg \min }\|\tilde{\boldsymbol{c}}\|_{1}, \quad \text { subject to } \quad\|\mathbf{\Psi} \tilde{\boldsymbol{c}}-\boldsymbol{u}\|_{2} \leq \varepsilon, \quad \mathbf{A A}^{\top}=\mathbf{I},
$$

where $\boldsymbol{\Psi}_{i j}=\Psi_{j}\left(\mathbf{A} \boldsymbol{\xi}^{i}\right)$. Here, the orthogonality requirement on $\mathbf{A}$ can be released to obtain a better result, i.e., making $\|\epsilon(\boldsymbol{\xi})\|_{2}$ smaller. However, even solving optimization problem $\left(P_{1, \varepsilon}^{R}\right)$ can be difficult when dimension is high, i.e., when $d$ is large, because the degree of freedom is $d+d(d-1)=d^{2}$. Similarly, the degree of freedom is $d+d^{2}=d(d+1)$ when the 
constraint $\mathbf{A} \mathbf{A}^{\top}=\mathbf{I}$ is not imposed. Therefore, we propose to use an alternating direction method that combines $\ell_{1}$ minimization solver and rotation matrix based on the gradients to approximate the solution of $\left(P_{1, \varepsilon}^{R}\right)$.

We start with an initial guess $u_{g}(\boldsymbol{\xi})=\sum_{n=1}^{N} \tilde{c}^{(0)} \psi_{n}(\boldsymbol{\xi})$, which is obtained by standard $\ell_{1}$ minimization or re-weighted $\ell_{1}$ minimization (Algorithm 2). Here, the superscript . $(l)$ stands for the $l$-th iteration, e.g., $\tilde{\boldsymbol{c}}^{(l)}$ are the gPC coefficients of the $l$-th iteration. We also set $\mathbf{A}^{(0)}=\mathbf{I}, \boldsymbol{\eta}^{(0)}=\boldsymbol{\xi}, v_{g}^{(0)}\left(\boldsymbol{\eta}^{(0)}\right)=u_{g}(\boldsymbol{\xi})$. In the $l$-th iteration $(l \geq 1)$, given $v_{g}^{(l-1)}$ and input samples $\left\{\left(\boldsymbol{\eta}^{(l-1)}\right)^{q}\right\}_{q=1}^{M}$, we first collect the gradient of $v_{g}^{(l-1)}$ :

$$
\mathbf{W}_{g}^{(l-1)}=\frac{1}{\sqrt{M}}\left[\nabla_{\xi} v_{g}^{(l-1)}\left(\left(\boldsymbol{\eta}^{(l-1)}\right)^{1}\right), \cdots, \nabla_{\xi} v_{g}^{(l-1)}\left(\left(\boldsymbol{\eta}^{(l-1)}\right)^{M}\right)\right],
$$

where $\nabla_{\xi^{*}}=\left(\partial \cdot / \partial \xi_{1}, \partial \cdot / \partial \xi_{2}, \cdots, \partial \cdot / \partial \xi_{d}\right)^{\top}$. Next, we compute the SVD of $\mathbf{W}_{g}^{(l-1)}$ :

$$
\mathbf{W}_{g}^{(l-1)}=\mathbf{U}_{W_{g}}^{(l-1)} \boldsymbol{\Sigma}_{W_{g}}^{(l-1)}\left(\mathbf{V}_{W_{g}}^{(l-1)}\right)^{\top}
$$

and set $\mathbf{A}^{(l)}=\left(\mathbf{U}_{W_{g}}^{(l-1)}\right)^{\top}$. Now, we can define a new set of random variables as $\boldsymbol{\eta}^{(l)}=$ $\mathbf{A}^{(l)} \boldsymbol{\xi}$ and compute their samples accordingly: $\left(\boldsymbol{\eta}^{(l)}\right)^{q}=\mathbf{A}^{(l)} \boldsymbol{\xi}^{q}$. We then construct a new measurement matrix $\boldsymbol{\Psi}^{(l)}$ as $\Psi_{i j}^{(l)}=\psi_{j}\left(\left(\boldsymbol{\eta}^{(l)}\right)^{i}\right)$, and solve the $\ell_{1}$ minimization problem $\left(P_{1, \varepsilon}\right)$ to obtain $\boldsymbol{c}^{(l)}$. Of note, the gradient in Eq. 24) is computed with respect to $\boldsymbol{\xi}$. Specifically, we use the chain rule in the computing:

$$
\begin{aligned}
& \nabla_{\xi} v_{g}^{(l)}\left(\left(\boldsymbol{\eta}^{(l)}\right)^{q}\right)=\nabla_{\xi} v_{g}^{(l)}\left(\mathbf{A}^{(l)} \boldsymbol{\xi}^{q}\right)=\left.\left(\mathbf{A}^{(l)}\right)^{\top} \nabla v_{g}^{(l)}(\boldsymbol{x})\right|_{\boldsymbol{x}=\mathbf{A}^{(l)} \xi^{q}} \\
= & \left.\left(\mathbf{A}^{(l)}\right)^{\top} \nabla \sum_{n=1}^{N} \tilde{c}_{n}^{(l)} \psi_{n}(\boldsymbol{x})\right|_{\boldsymbol{x}=\mathbf{A}^{(l)} \xi^{q}}=\left.\left(\mathbf{A}^{(l)}\right)^{\top} \sum_{n=1}^{N} \tilde{c}_{n}^{(l)} \nabla \psi_{n}(\boldsymbol{x})\right|_{\boldsymbol{x}=\mathbf{A}^{(l)} \xi^{q}} .
\end{aligned}
$$

Therefore, we only need to evaluate $\nabla \psi_{n}$ at $\left(\boldsymbol{\eta}^{(l)}\right)^{q}$. This is straightforward because we construct $\psi_{n}$ using the tensor product of univariate polynomials (Eq. (5)) and derivatives of widely used orthogonal polynomials in UQ study, e.g., Hermite, Laguerre, Legendre and Chebyshev, are known. We summarize the entire procedure in Algorithm 3. The stopping criterion we use is the difference between to successive rotation matrix $\mathbf{A}^{(l)}$ and $\mathbf{A}^{(l+1)}$. The threshold $\theta$ can be set as a fraction of the dimension, e.g., $0.2 d$. Alternatively, it can be set as $l \leq l_{\max }$, where $l_{\max }$ is can taken as 2 or 3 empirically for practical problems. Because it is not necessary to use the optimal rotation to improve the sparsity. In addition, the sparsity structure is problem-dependent (see examples in Section 44), and, for many practical problems, the improvement is not made significant by using more iterations. Notably, we update $\boldsymbol{c}^{(l)}$ and $\mathbf{A}^{(l)}$ separately in each iteration, which is the spirit of the alternating direction method.

\subsection{A comparison with the Gaussian case}

An alternating direction algorithm approximating the solution of $\left(P_{1, \epsilon}^{R}\right)$ when $\xi_{i}$ are i.i.d. Gaussian random variables, i.e., $\psi_{n}$ are Hermite polynomials, was proposed in 56, and we present it in Algorithm 4. In this section, we illustrate the connections and differences between Algorithms 3 and 4

These algorithms use different approaches to obtain rotation matrix that maps $\boldsymbol{\xi}$ to $\boldsymbol{\eta}$. Both of them use gradient information. However, Algorithm 3 stems from SVD of $\mathbf{W}$ in Eq. (17), while Algorithm 4 roots on eigendecomposition of the variance of the gradients in Eq. (15) as in 30. In particular, Steps 5-8 of Algorithm 4 is a more sophisticated version of Algorithm 1 in 30. (because $\mathbf{G}$ is approximated with an analytic form), which can be considered as a special case of Algrothm 4 without iteration. When $u$ is known, they are equivalent asymptotically as proven in Lemmas (3.1) and 3.2. Although, in practice, 
Algorithm 3 Alternating direction method of solving $\left(P_{1, \epsilon}^{R}\right)$

1: Generate input samples $\left\{\boldsymbol{\xi}^{q}\right\}_{q=1}^{M}$ based on the distribution of $\boldsymbol{\xi}$.

2: Generate corresponding output samples $\left\{u^{q}=u\left(\boldsymbol{\xi}^{q}\right)\right\}_{q=1}^{M}$ by solving the complete model, e.g., running simulations, solvers, etc.

3: Select gPC basis functions $\left\{\psi_{n}\right\}_{n=1}^{N}$ associated with $\boldsymbol{\xi}$ and then generate the measurement matrix $\boldsymbol{\Psi}$ by setting $\Psi_{i j}=\psi_{j}\left(\boldsymbol{\xi}^{i}\right)$.

4: Solve the optimization problem $\left(P_{1, \epsilon}\right)$ :

$$
\arg \min _{\hat{\boldsymbol{c}}}\|\hat{\boldsymbol{c}}\|_{1}, \text { subject to }\|\mathbf{\Psi} \hat{\boldsymbol{c}}-\boldsymbol{u}\|_{2} \leq \epsilon .
$$

5: Set counter $l=0, \boldsymbol{\eta}^{(0)}=\boldsymbol{\xi}, \tilde{\boldsymbol{c}}^{(0)}=\hat{\boldsymbol{c}}, \mathbf{A}^{(0)}=\mathbf{I}$, and $v_{g}^{(0)}=\sum_{n=1}^{N} \tilde{c}^{(0)} \psi_{n}(\boldsymbol{\xi})$.

6: $l=l+1$. Construct $\mathbf{W}_{g}^{(l-1)}$ in Eq. 24) by computing $\nabla_{\xi} v_{g}^{(l-1)}\left(\left(\boldsymbol{\eta}^{(l-1)}\right)^{q}\right)$ according to Eq. (26). Then compute SVD of $\mathbf{W}_{g}^{(l)}: \mathbf{W}_{g}^{(l)}=\mathbf{U}_{W_{g}}^{(l)} \boldsymbol{\Sigma}_{W_{g}}^{(l)}\left(\mathbf{V}_{W_{g}}^{(l)}\right)^{\top}$.

7: Set $\mathbf{A}^{(l)}=\left(\mathbf{U}_{W_{g}}^{(l)}\right)^{\top}$ and $\boldsymbol{\eta}^{(l)}=\mathbf{A}^{(l)} \boldsymbol{\xi}$. Then compute samples $\left(\boldsymbol{\eta}^{(l)}\right)^{q}=\mathbf{A}^{(l)} \boldsymbol{\xi}^{q}, q=$ $1,2, \cdots, M$. Construct the new measurement matrix $\Psi^{(l)}$ with $\Psi_{i j}^{(l)}=\psi_{j}\left(\left(\boldsymbol{\eta}^{(l)}\right)^{i}\right)$.

8: Solve the optimization problem $\left(P_{1, \epsilon^{(l)}}\right)$ :

$$
\arg \min _{\hat{\boldsymbol{c}}}\|\hat{\boldsymbol{c}}\|_{1}, \quad \text { subject to }\left\|\Psi^{(l)} \hat{\boldsymbol{c}}-\boldsymbol{u}\right\|_{2} \leq \epsilon^{(l)},
$$

then set $\tilde{\boldsymbol{c}}^{(l)}=\hat{\boldsymbol{c}}$ and $v_{g}^{(l)}\left(\boldsymbol{\eta}^{(l)}\right)=\sum_{n=1}^{N} \tilde{c}^{(l)} \psi_{n}\left(\boldsymbol{\eta}^{(l)}\right)$.

9: If $\left\|\mathbf{A}^{(l)}-\mathbf{A}^{(l-1)}\right\|_{2}<\theta$, where the threshold $\theta$ is a positive real number, then stop. Otherwise, go to Step 6.

10: Construct gPC expansion as $u(\boldsymbol{\xi}) \approx u_{g}(\boldsymbol{\xi})=v_{g}\left(\boldsymbol{\eta}^{(l)}\right)=\sum_{n=1}^{N} \tilde{c}_{n}^{(l)} \psi_{n}\left(\mathbf{A}^{(l)} \boldsymbol{\xi}\right)$.

$u$ is unknown and $M$ is limited, these lemmas still provide intuitive understanding of the connection between Algorithm 3 and Algorithm 4 they both use the distinct importance of subspaces identified by $\nabla u$ to enhance the sparsity via projecting $\boldsymbol{\xi}$ to these subspaces.

For the Gaussian case, i.e., Algorithm 4 , we use Eq. (15) and approximate $\mathbf{G}$ as

$$
\mathbf{G} \approx \mathbb{E}\left\{\nabla u_{g}(\boldsymbol{\xi}) \cdot \nabla u_{g}(\boldsymbol{\xi})^{\top}\right\}=\mathbb{E}\left\{\nabla\left(\sum_{n=1}^{N} c_{n} \psi_{n}(\boldsymbol{\xi})\right) \cdot \nabla\left(\sum_{n^{\prime}=1}^{N} c_{n^{\prime}} \psi_{n^{\prime}}(\boldsymbol{\xi})\right)^{\top}\right\} .
$$

For simplicity, we denote $\frac{\partial h(\boldsymbol{x})}{\partial x_{i}}$ as $\partial_{i} h(\boldsymbol{x})$ for any differentiable function $h$. Then, Eq. 28 implies that

$$
\begin{aligned}
G_{i j} & \approx \mathbb{E}\left\{\partial_{i}\left(\sum_{n=1}^{N} c_{n} \psi_{n}(\boldsymbol{\xi})\right) \cdot \partial_{j}\left(\sum_{n^{\prime}=1}^{N} c_{n^{\prime}} \psi_{n^{\prime}}(\boldsymbol{\xi})\right)\right\}=\mathbb{E}\left\{\left(\sum_{n=1}^{N} c_{n} \partial_{i} \psi_{n}(\boldsymbol{\xi})\right) \cdot\left(\sum_{n^{\prime}=1}^{N} c_{n^{\prime}} \partial_{j} \psi_{n^{\prime}}(\boldsymbol{\xi})\right)\right\} \\
& =\sum_{n=1}^{N} \sum_{n^{\prime}=1}^{N} c_{n} c_{n^{\prime}} \mathbb{E}\left\{\partial_{i} \psi_{n}(\boldsymbol{\xi}) \cdot \partial_{j} \psi_{n^{\prime}}(\boldsymbol{\xi})\right\}=\boldsymbol{c}^{T} \mathbf{K}_{i j} \boldsymbol{c} .
\end{aligned}
$$

In each iteration of Algorithm 4 ,

$$
G_{i j}^{(l)}=\mathbb{E}\left\{\partial_{i}\left(\sum_{n=1}^{N} \tilde{c}_{n}^{(l-1)} \psi_{n}\left(\boldsymbol{\eta}^{(l-1)}\right)\right) \cdot \partial_{j}\left(\sum_{n^{\prime}=1}^{N} \tilde{c}_{n^{\prime}}^{(l-1)} \psi_{n^{\prime}}\left(\boldsymbol{\eta}^{(l-1)}\right)\right)\right\}=\left(\tilde{\boldsymbol{c}}^{(l-1)}\right)^{\top} \mathbf{K}_{i j}^{(l-1)} \tilde{\boldsymbol{c}}^{(l-1)},
$$


Algorithm 4 Alternating direction algorithm for $\left(P_{1, \varepsilon}^{R}\right)$ when $\xi_{i}$ are i.i.d. Gaussian.

1: Generate input samples $\left\{\boldsymbol{\xi}^{q}\right\}_{q=1}^{M}$ based on the distribution of $\boldsymbol{\xi}$.

2: Generate output samples $\left\{u^{q}=u\left(\boldsymbol{\xi}^{q}\right)\right\}_{q=1}^{M}$ by solving the complete model.

3: Set $\left\{\psi_{n}\right\}_{n=1}^{N}$ as multivariate Hermite polynomials, then generate the measurement matrix $\boldsymbol{\Psi}$ as $\Psi_{i j}=\psi_{j}\left(\boldsymbol{\xi}^{i}\right)$.

4: Solve the optimization problem $\left(P_{1, \epsilon}\right)$ :

$$
\arg \min _{\hat{\boldsymbol{c}}}\|\hat{\boldsymbol{c}}\|_{1}, \text { subject to }\|\boldsymbol{\Psi} \hat{\boldsymbol{c}}-\boldsymbol{u}\|_{2} \leq \epsilon .
$$

5: Set counter $l=0, \eta^{(0)}=\boldsymbol{\xi}, \tilde{\boldsymbol{c}}^{(0)}=\hat{\boldsymbol{c}}$, and compute $\mathbf{K}_{i j}, i, j=1,2, \cdots, N$ as

$$
\left(K_{i j}\right)_{k m}=\mathbb{E}\left\{\frac{\partial \psi_{k}}{\partial x_{i}}(\boldsymbol{\xi}) \cdot \frac{\partial \psi_{m}}{\partial x_{j}}(\boldsymbol{\xi})\right\} .
$$

6: $l=l+1$. Construct $\mathbf{G}^{(l-1)}$ as $G_{i j}^{(l)}=\left(\tilde{\boldsymbol{c}}^{(l-1)}\right)^{\top} \mathbf{K}_{i j} \tilde{\boldsymbol{c}}^{(l-1)}, i, j=1,2, \cdots, d$. Then, compute eigendecomposition of $\mathbf{G}^{(l-1)}: \mathbf{G}^{(l-1)}=\mathbf{U}^{(l-1)} \boldsymbol{\Lambda}^{(l-1)}\left(\mathbf{U}^{(l-1)}\right)^{\top}$.

7: Set $\boldsymbol{\eta}^{(l)}=\left(\mathbf{U}^{(l)}\right)^{\top} \boldsymbol{\eta}^{(l-1)}$, then compute samples $\left(\boldsymbol{\eta}^{(l)}\right)^{q}=\left(\mathbf{U}^{(l-1)}\right)^{\top}\left(\boldsymbol{\eta}^{(l-1)}\right)^{q}, q=$ $1,2, \cdots, M$. Construct the new measurement matrix $\Psi^{(l)}$ with $\Psi_{i j}^{(l)}=\psi_{j}\left(\left(\boldsymbol{\eta}^{(l)}\right)^{i}\right)$.

8: Solve the optimization problem $\left(P_{1, \epsilon(l)}\right)$ :

$$
\arg \min _{\hat{\boldsymbol{c}}}\|\hat{\boldsymbol{c}}\|_{1}, \quad \text { subject to }\left\|\Psi^{(l)} \hat{\boldsymbol{c}}-\boldsymbol{u}\right\|_{2} \leq \epsilon^{(l)},
$$

and set $\tilde{\boldsymbol{c}}^{(l)}=\hat{\boldsymbol{c}}$.

9: If $\left|\left\|\widetilde{\mathbf{U}}^{(l)}\right\|_{1}-d\right|<\theta$, where the threshold $\theta$ is a positive real number, then stop. Otherwise, go to Step 6 .

10: Set

$$
\mathbf{A}^{(l)}=\left(\mathbf{U}^{(0)} \mathbf{U}^{(2)} \cdots \mathbf{U}^{(l-1)}\right)^{\top}
$$

and construct gPC expansion as $u(\boldsymbol{\xi}) \approx u_{g}(\boldsymbol{\xi})=v_{g}\left(\boldsymbol{\eta}^{(l)}\right)=\sum_{n=1}^{N} \tilde{c}_{n}^{(l)} \psi_{n}\left(\mathbf{A}^{(l)} \boldsymbol{\xi}\right)$.

and

$$
\left(K_{i j}^{(l-1)}\right)_{k m}=\mathbb{E}\left\{\partial_{i} \psi_{k}\left(\boldsymbol{\eta}^{(l-1)}\right) \cdot \partial_{j} \psi_{m}\left(\boldsymbol{\eta}^{(l-1)}\right)\right\} .
$$

In this instance, we do not need to update $\mathbf{K}_{i j}$ because $\eta_{i}^{(l)}$ are i.i.d. Gaussian for each $l$, and

$$
\mathbb{E}\left\{\partial_{i} \psi_{k}\left(\boldsymbol{\eta}^{(l)}\right) \cdot \partial_{j} \psi_{m}\left(\boldsymbol{\eta}^{(l)}\right)\right\} \equiv \frac{1}{(2 \pi)^{d / 2}} \int_{\mathbb{R}^{d}} \partial_{i} \psi_{k}(\boldsymbol{x}) \cdot \partial_{j} \psi_{m}(\boldsymbol{x}) \exp \left(-\frac{\|\boldsymbol{x}\|_{2}^{2}}{2}\right) \mathrm{d} \boldsymbol{x}
$$

are fixed so it can be precomputed (see [56]). The Algorithms 4 s design employs this advantage. However, for general cases, this good property may not exist, and we need to update $\mathbf{K}_{i j}$ in each step if we use Eq. (29). Specifically, in the $l$-th iteration of Algorithm 3 .

$$
\left|\operatorname{det}\left(\frac{D \boldsymbol{\eta}^{(l-1)}}{D \boldsymbol{\xi}}\right)\right|=\left|\operatorname{det}\left(\left(\mathbf{A}^{(l-1)}\right)^{-1}\right)\right|=1,
$$

and the PDF of $\boldsymbol{\eta}^{(l-1)}$ is

$$
\rho_{\boldsymbol{\eta}^{(l-1)}}(\boldsymbol{x})=\rho_{\boldsymbol{\xi}}\left(\left(\mathbf{A}^{(l-1)}\right)^{-1} \boldsymbol{x}\right)\left|\operatorname{det}\left(\frac{D \boldsymbol{\xi}}{D \boldsymbol{\eta}^{(l-1)}}\right)\right|=\rho_{\boldsymbol{\xi}}\left(\left(\mathbf{A}^{(l-1)}\right)^{-1} \boldsymbol{x}\right) .
$$


Thus, if we want to approximate $\mathbf{G}$ as in Algorithm 4 , we need to compute

$$
\begin{aligned}
\mathbb{E}\left\{\partial_{i} \psi_{n}\left(\boldsymbol{\eta}^{(l-1)}\right) \cdot \partial_{j} \psi_{n^{\prime}}\left(\boldsymbol{\eta}^{(l-1)}\right)\right\} & =\int_{\Omega_{\boldsymbol{\eta}^{(l-1)}}} \partial_{i} \psi_{n}(\boldsymbol{x}) \cdot \partial_{j} \psi_{n^{\prime}}(\boldsymbol{x}) \rho_{\boldsymbol{\eta}^{(l-1)}}(\boldsymbol{x}) \mathrm{d} \boldsymbol{x} \\
& =\int_{\Omega_{\boldsymbol{\eta}^{(l-1)}}} \partial_{i} \psi_{n}(\boldsymbol{x}) \cdot \partial_{j} \psi_{n^{\prime}}(\boldsymbol{x}) \rho_{\boldsymbol{\xi}}\left(\left(\mathbf{A}^{(l-1)}\right)^{-1} \boldsymbol{x}\right) \mathrm{d} \boldsymbol{x},
\end{aligned}
$$

where $\Omega_{\boldsymbol{\eta}^{(l-1)}}$ is the domain of multivariate random variable $\boldsymbol{\eta}^{(l-1)}$. If $\boldsymbol{\xi} \sim \mathcal{N}(\mathbf{0}, \mathbf{I})$, then $\Omega_{\boldsymbol{\eta}^{(l-1)}}=\Omega_{\boldsymbol{\xi}}$, and

$$
\rho_{\boldsymbol{\eta}^{(l-1)}}(\boldsymbol{x})=\rho_{\boldsymbol{\xi}}\left(\left(\mathbf{A}^{(l-1)}\right)^{-1} \boldsymbol{x}\right)=\rho_{\boldsymbol{\xi}}(\boldsymbol{x}) .
$$

Hence, we only need to compute $\mathbf{K}_{i j}$ once. In other cases, updating $\mathbf{K}_{i j}$ in each iteration can be costly because of the high-dimensional integral in Eq. (34).

A possible solution is to compute $\mathbf{K}_{i j}$ based on $\rho_{\boldsymbol{\xi}}$ first. Then, in each iteration, after obtaining $v_{g}^{(l)}\left(\boldsymbol{\eta}^{(l)}\right)=\sum_{i=1}^{N} \tilde{c}_{n}^{(l)} \psi_{n}\left(\boldsymbol{\eta}^{(l)}\right)$, we compute the corresponding $u_{g}^{(l)}(\boldsymbol{\xi})=$ $\sum_{i=1}^{N} c_{n}^{(l)} \psi_{n}(\boldsymbol{\xi})$ through algebraic computing or by accurate numerical integral:

$$
c_{n}^{(l)}=\int_{\Omega_{\boldsymbol{\xi}}} v_{g}^{(l)}\left(\mathbf{A}^{(l)} \boldsymbol{x}\right) \psi_{n}(\boldsymbol{x}) \rho_{\boldsymbol{\xi}}(\boldsymbol{x}) \mathrm{d} \boldsymbol{x}=\sum_{q=1}^{N_{q}} v_{g}^{(l)}\left(\mathbf{A}^{(l)} \boldsymbol{x}^{q}\right) \psi_{n}\left(\boldsymbol{x}^{q}\right) w^{q},
$$

where $\boldsymbol{x}^{q}$ and $w^{q}$ are quadrature points and weights with respect to $\rho_{\boldsymbol{\xi}}(\boldsymbol{x})$. As such, $G_{i j}^{(l)}$ can be approximated by $\left(\boldsymbol{c}^{l}\right)^{T} \mathbf{K}_{i j} \boldsymbol{c}^{(l)}$. However, the additional computation cost for converting $v_{g}^{(l)}$ to $u_{g}^{(l)}$ makes this algorithm less efficient. Therefore, we choose to use the SVD of $\mathbf{W}_{g}$ in Algorithm 3

In addition to the different approaches for computing the rotation matrix, another difference is that in each iteration, Algorithm 3 directly identifies rotation matrix $\mathbf{A}^{(l)}$ that maps $\boldsymbol{\xi}$ to $\boldsymbol{\eta}^{(l)}$, while Algorithm 4 seeks for $\mathbf{U}^{(l)}$, which is a "correction" of the existing rotation, that maps $\boldsymbol{\eta}^{(l-1)}$ to $\boldsymbol{\eta}^{(l)}$. In each iteration of Algorithm 4 we do not need to compute $\mathbf{A}^{(l)}$, and we compute it after the iterations terminate. Thus, Algorithm 4 does not rely on the chain rule. We also can design Algorithm 3 in this manner, i.e., each iteration maps $\boldsymbol{\eta}^{(l-1)}$ to $\boldsymbol{\eta}^{(l)}$. Instead, we use the current design to explicitly fit the description of the alternating direction method, i.e., in each iteration, we explicitly identify $\tilde{\boldsymbol{c}}^{(l)}$ and $\mathbf{A}^{(l)}$ separately.

Finally, according to our numerical tests (not shown), there is no significant difference between the accuracy of Algorithms 3 and 4 for Hermite polynomial expansions. This indicates that the general framework in Algorithm 3 also is efficient for $u$ relying on i.i.d. Gaussian random variables.

\subsection{Compromising the property of $\Psi$}

We already have discussed the possible enhancement of the sparsity in $\tilde{\boldsymbol{c}}$ by introducing the rotation. However, as we indicated at the beginning of this section, $\left\{\psi_{n}\right\}_{n=1}^{N}$ are not necessarily orthonormal to each other with respect to $\rho_{\boldsymbol{\eta}}$. The property of matrix $\boldsymbol{\Psi}^{(l)}$ may become less favorable for the $\ell_{1}$ minimization. The most straightforward conclusion we can obtain is that the mutual coherence of $\boldsymbol{\Psi}^{(l)}(l \geq 1)$ can be larger than that of $\boldsymbol{\Psi}^{(0)}$. Here, the mutual coherence [6], defined as

$$
\mu(\boldsymbol{\Psi}) \stackrel{\text { def }}{=} \max _{1 \leq j, k \leq N, j \neq k} \frac{\left|\boldsymbol{\Psi}_{j}^{T} \mathbf{\Psi}_{k}\right|}{\left\|\boldsymbol{\Psi}_{j}\right\|_{2} \cdot\left\|\boldsymbol{\Psi}_{k}\right\|_{2}},
$$

where $\boldsymbol{\Psi}_{j}$ and $\boldsymbol{\Psi}_{k}$ are columns of $\boldsymbol{\Psi}$, is a more tractable property of the measurement matrix than the RIP. Generally, a measurement matrix with smaller mutual coherence is better able to recover a sparse solution with the compressive sensing method. When $\psi_{n}$ are Hermite polynomials ( $\left\{\xi_{i}\right\}_{i=1}^{d}$ are i.i.d. Gaussian), $\mu(\boldsymbol{\Psi})$ is conserved (statistically) because $\boldsymbol{\eta}^{(l)}$ are still i.i.d. Gaussian. This adds to the specialty of the Hermite polynomial expansions. For other polynomials, the aforementioned rotational method can be less efficient in some 
cases due to the increase of $\mu(\boldsymbol{\Psi})$ (detailed in Section 4). We note that in the compressive sensing theory, the number of samples needed for an accurate computing of $\boldsymbol{c}$ is related to both the sparsity of $\boldsymbol{c}$ and the property of $\boldsymbol{\Psi}$. Therefore, for general cases, although we can increase the sparsity of $\boldsymbol{c}$, if $\boldsymbol{\Psi}$ becomes worse (i.e., it loses RIP or $\mu$ increases), out method's efficiency can be affected.

\section{Numerical Examples}

In this section, we revisit the five numerical examples in 56] with different types of random variables in the systems and various polynomial expansions to approximate the solution. By testing the same examples (with different types of random variables), we demonstrate the efficiency of the proposed general framework and we can compare the performance with the special case (Hermite polynomial expansion) in our previous study. Specifically, the random variables considered in this section are uniform random variables $\mathcal{U}[-1,1]^{d}$ (associated with Legendre polynomials) and Chebyshev random variables with $\operatorname{PDF} \rho(\boldsymbol{x})=\left(\frac{1}{\pi \sqrt{1-x^{2}}}\right)^{d}$ (associated with Chebyshev polynomials of the first kind, which is denoted as Chebyshev polynomial for simplicity). The accuracies of different methods are measured by the relative $L_{2}$ error: $\left(\left\|u-u_{g}\right\|_{2}\right) /\|u\|_{2}$, where $u_{g}$ is the Legendre polynomial expansion or Chebyshev polynomial expansion of $u$. The integral

$$
\|u(\boldsymbol{\xi})\|_{2}=\left(\int_{\mathbb{R}^{d}} u(\boldsymbol{\xi})^{2} \rho(\boldsymbol{\xi}) \mathrm{d} \boldsymbol{\xi}\right)^{1 / 2}
$$

(and $\left\|u-u_{g}\right\|_{2}$ ) is approximated with a high-level sparse grids method, based on onedimensional Gaussian quadrature and the Smolyak structure [42. The term "level" $p$ means that the algebraic accuracy of the sparse grids method is $2 p-1$. We use $P$ to denote the truncation order, which implies that polynomials up to order $P$ are included in expansion $u_{g}$. Hence, the number of unknowns can be computed as $N=\left(\begin{array}{c}P+d \\ d\end{array}\right)$.

The relative errors we present in this section are obtained from 50 independent replicates for each sample size $M$. For example, we generate 50 independent sets of input samples $\boldsymbol{\xi}^{q}, q=1,2, \cdots, M$, compute 50 different relative errors, and report the average of these error samples. To investigate the effectiveness of increasing the output samples, we set the $x$-axis in our figures as the ratio $M / N$, which is the fraction of available data with respect to number of unknowns. We use MATLAB package SPGL1 [48, 47] to solve $\left(P_{1, \epsilon}\right)$. If not otherwise indicated, we use Algorithm 3 , and results are obtained with $l_{\max }=3$ iterations. We use three iterations in the re-weighted $\ell_{1}$ minimization to solve $\left(P_{1, \epsilon}\right)$.

\subsection{Ridge function}

Consider the following ridge function:

$$
u(\boldsymbol{\xi})=\sum_{i=1}^{d} \xi_{i}+0.25\left(\sum_{i=1}^{d} \xi_{i}\right)^{2}+0.025\left(\sum_{i=1}^{d} \xi_{i}\right)^{3}
$$

where all $\xi_{i}$ are equally important. In this case, adaptive methods that build the surrogate model hierarchically based on the importance of $\xi_{i}$ (e.g., [33, 54, 60]) may not be efficient. A simple rotation matrix for this example has the form

$$
\mathbf{A}=\left(\begin{array}{rrrr}
d^{-1 / 2} & d^{-1 / 2} & \cdots & d^{-1 / 2} \\
& \tilde{\mathbf{A}} & & \\
& &
\end{array}\right),
$$

where $\tilde{\mathbf{A}}$ is a $(d-1) \times d$ matrix chosen to ensure that $\mathbf{A}$ is orthonormal. Given this choice for $\mathbf{A}, \eta_{1}=\left(\sum_{i=1}^{d} \xi_{i}\right) / d^{1 / 2}$ and $u$ has a very simple representation:

$$
u(\boldsymbol{\xi})=v(\boldsymbol{\eta})=d^{1 / 2} \eta_{1}+0.25 d \eta_{1}^{2}+0.025 d^{3 / 2} \eta_{1}^{3} .
$$


Therefore, as we keep the set of the basis functions unchanged, all of the polynomials not related to $\eta_{1}$ make no contribution to the expansion, which implies that we obtain a very sparse representation of $u$. Becasue the optimal structure is not known a priori, the standard compressive sensing cannot take advantage of it.

In this test, we set $d=12$ (hence, $N=455$ for $P=3$ ) and demonstrate the effectiveness of our new method. The integrals for calculating the $L_{2}$ error are computed by a level 4 sparse grids method. Therefore, they are exact. The relative errors are presented in Figure 1 for Legendre polynomial expansion (assuming $\xi_{i}$ are i.i.d. uniform random variables) and Chebyshev polynomial expansion (assuming $\xi_{i}$ are i.i.d. Chebyshev random variables). Clearly, the standard $\ell_{1}$ minimization is not effective as the relative error is close to $50 \%$ even when $M / N$ approaches 0.4 . Also, the re-weighted $\ell_{1}$ does not help in this case. However, our new iterative rotation demonstrates much better accuracy, especially when $M$ is large. We notice that the accuracy increases as more iterations are included. Moreover, the improvement from six iterations to nine iterations is less significant as that from three iterations to six iterations because the rotation detected by the algorithm approaches the optimal one.
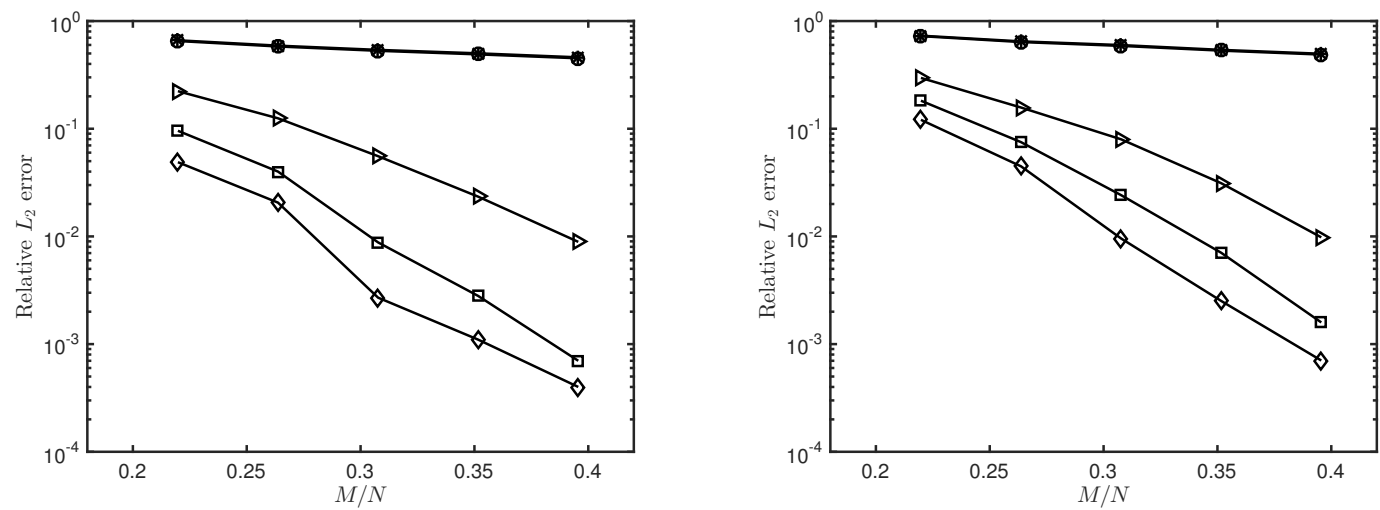

Figure 1: Results for the ridge function. Left: Legendre polynomial expansion (when $\xi_{i}$ are i.i.d. uniform random variables); Right: Chebyshev polynomial expansion (when $\xi_{i}$ are i.i.d. Chebyshev random variables). "o": standard $\ell_{1}$, "*": re-weighted $\ell_{1}$, " $": \ell_{1}$ with three rotations, " $\square$ ": $\ell_{1}$ with six rotations, " $\diamond ": \ell_{1}$ with nine rotations.

Figure 2 compares the absolute values of exact coefficients $c_{n}$ and the coefficients $\tilde{c}_{n}$ after nine iterations using 180 samples. In this figure we, do not present $\tilde{c}_{n}$ with absolute value smaller than $10^{-3}$ because they are more than two magnitudes smaller than the dominating ones. As demonstrated in Figure 2, the iterative rotation creates a much sparser representation of $u$. Thus, the efficiency of compressive sensing method is substantially enhanced. Notably, this is a special example in that ridge function has very good low-dimensional structure (it is a one-dimensional function after an appropriate linear transform). In general, many systems does not have this ideal structure, and the improvement afforded by iterative rotations usually diminishes after two to three iterations.

\subsection{Function with high compressibility}

Consider the following function:

$$
u(\boldsymbol{\xi})=\sum_{|\boldsymbol{\alpha}|=0}^{P} c_{\boldsymbol{\alpha}} \psi_{\boldsymbol{\alpha}}(\boldsymbol{\xi})=\sum_{n=1}^{N} c_{n} \psi_{n}(\boldsymbol{\xi}), \quad \boldsymbol{\xi}=\left(\xi_{1}, \xi_{2}, \cdots, \xi_{d}\right)^{\top},
$$

where, $\psi_{\boldsymbol{\alpha}}$ are normalized multivariate Legendre or Chebyshev polynomials, $d=12, P=$ $3, N=455$, and the coefficients $c_{n}$ are chosen as uniformly distributed random numbers,

$$
c_{n}=\zeta / n^{1.5}, \quad \zeta \sim \mathcal{U}[0,1] .
$$




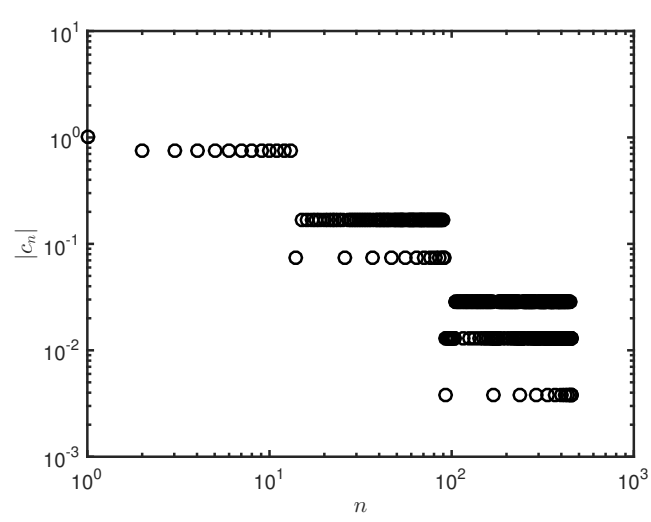

(a) Legendre $\left|c_{n}\right|$

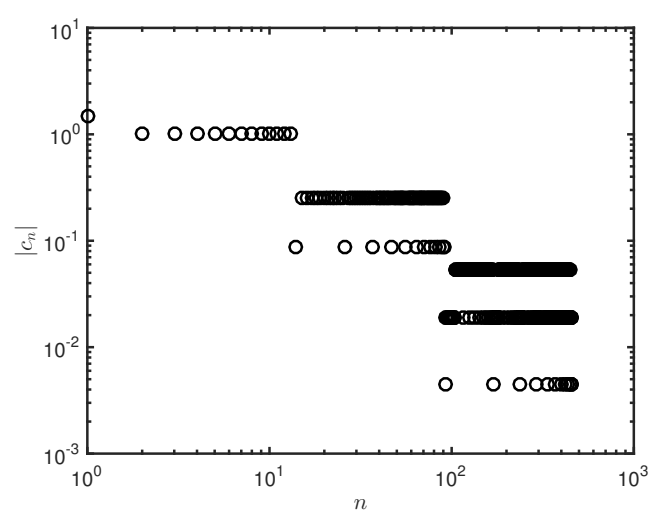

(c) Chebyshev $\left|c_{n}\right|$

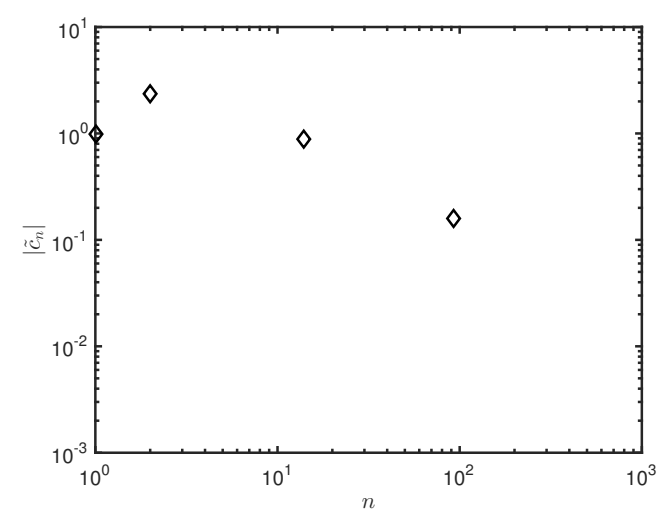

(b) Legendre $\left|\tilde{c}_{n}\right|$

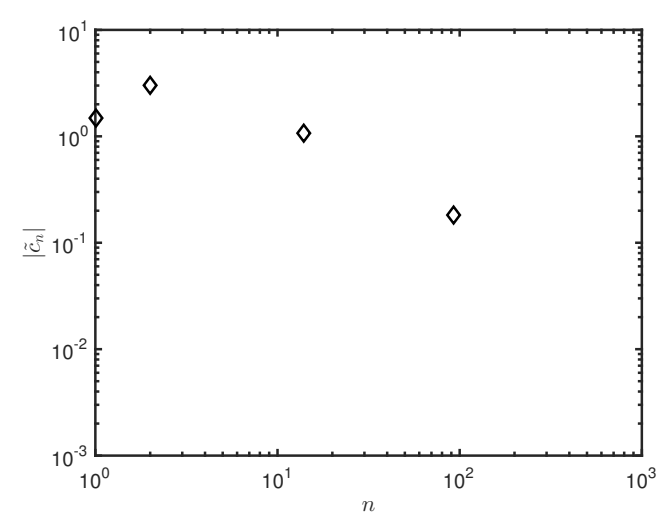

(d) Chebyshev $\left|\tilde{c}_{n}\right|$

Figure 2: Results for the ridge function. Absolute values of exact coefficients $c_{n}$ and coefficients $\tilde{c}_{n}$ after rotations using 180 samples.

For this example, we generate $N$ samples of $\zeta: \zeta^{1}, \zeta^{2}, \cdots, \zeta^{N}$ then divide them by $n^{1.5}, n=$ $1,2, \cdots, N$ to obtain a random "compressible signal" $\boldsymbol{c}$. The integrals for the relative error are computed by a level- 4 sparse grid method and, therefore, are exact. Figure 3 shows the relative $L_{2}$ errors obtained by applying our iterative rotation technique to the re-weighted $\ell_{1}$ approach. Apparently, introduction of the iterative rotation approach improves the accuracy. Figure 4 presents a comparison of the absolute values of entries of $\boldsymbol{c}$ and $\tilde{\boldsymbol{c}}$ (using 160 samples). The main improvement is that the number of coefficients with magnitudes larger than 0.01 decreased. Also, $c_{n}$ cluster around the curve $c_{n}=1 / n^{1.5}$ as we set them in this way, while many $\tilde{c}_{n}$ appear below this curve, especially when $n$ is large. In Figure 4. we also compare the values of $\frac{\left\|\boldsymbol{c}-\boldsymbol{c}_{s}\right\|_{1}}{\sqrt{s}}$ and $\frac{\left\|\tilde{\boldsymbol{c}}-\tilde{\boldsymbol{c}}_{s}\right\|_{1}}{\sqrt{s}}$ to demonstrate quantitatively the enhancement of the sparsity after rotations.

\subsection{Elliptic equation}

Next, we consider a one-dimensional elliptic differential equation with a random coefficient:

$$
\begin{aligned}
-\frac{d}{d x}\left(a(x ; \boldsymbol{\xi}) \frac{d u(x ; \boldsymbol{\xi})}{d x}\right) & =1, \quad x \in(0,1) \\
u(0)=u(1) & =0
\end{aligned}
$$



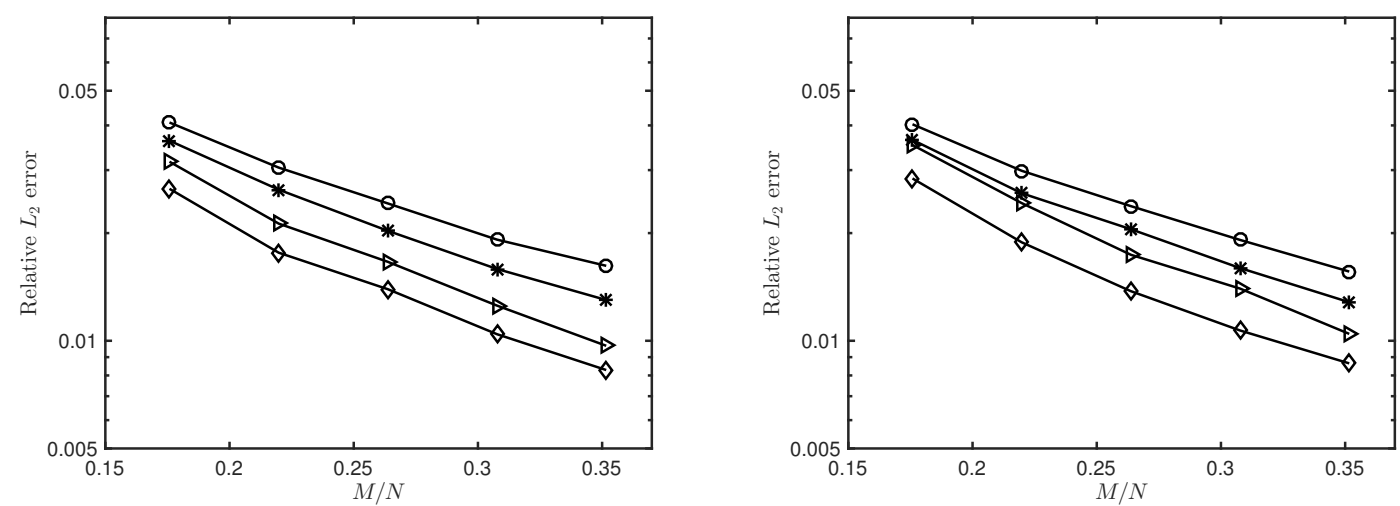

Figure 3: Results for the highly compressible function. Left: Legendre polynomial expansion (when $\xi_{i}$ are i.i.d. uniform random variables). Right: Chebyshev polynomial expansion (when $\xi_{i}$ are i.i.d. Chebyshev random variables). "o": standard $\ell_{1}$, "*": re-weighted $\ell_{1}$, "ฉ": rotated $\ell_{1}$, "»": re-weighted+rotated $\ell_{1}$.

where $a(x ; \boldsymbol{\xi})$ is a log-normal random field based on Karhunen-Loève (KL) expansion:

$$
a(x ; \boldsymbol{\xi})=a_{0}(x)+\exp \left(\sigma \sum_{i=1}^{d} \sqrt{\lambda_{i}} \phi_{i}(x) \xi_{i}\right),
$$

where $\left\{\xi_{i}\right\}$ are i.i.d. random variables, $\left\{\lambda_{i}\right\}_{i=1}^{d}$, and $\left\{\phi_{i}(x)\right\}_{i=1}^{d}$ are the largest eigenvalues and corresponding eigenfunctions of the exponential covariance kernel:

$$
C\left(x, x^{\prime}\right)=\exp \left(-\frac{\left|x-x^{\prime}\right|}{l_{c}}\right) .
$$

In the KL expansion, $\lambda_{i}$ denotes the eigenvalue of the covariance kernel $C\left(x, x^{\prime}\right)$ instead of entries of $\Lambda$ in Eq. (29). The value of $\lambda_{i}$ and the analytical expressions for $\phi_{i}$ are available in the literature 27$]$. In this example, we set $a_{0}(x) \equiv 0.1, \sigma=0.5, l_{c}=0.2$, and $d=15$. With this setting, $\sum_{i=1}^{d} \lambda_{i}>0.93 \sum_{i=1}^{\infty} \lambda_{i}$. For each input sample $\boldsymbol{\xi}^{q}, a$ and $u$ only depend on $x$, and the solution of the deterministic elliptic equation can be obtained as [55]:

$$
u(x)=u(0)+\int_{0}^{x} \frac{a(0) u(0)^{\prime}-y}{a(y)} \mathrm{d} y .
$$

By imposing the boundary condition $u(0)=u(1)=0$, we can compute $a(0) u(0)^{\prime}$ as

$$
a(0) u(0)^{\prime}=\left(\int_{0}^{1} \frac{y}{a(y)} \mathrm{d} y\right) /\left(\int_{0}^{1} \frac{1}{a(y)} \mathrm{d} y\right) .
$$

The integrals in Eqs. (46) and (45) are obtained by highly accurate numerical integration. For this example, we choose the QoI to be $u(x ; \boldsymbol{\xi})$ at $x=0.35$. We aim to build a third-order Legendre (or Chebyshev) polynomial expansion that includes $N=816$ basis functions. The relative error is approximated by a level-6 sparse grid method. Figure 5 shows that accuracy of the re-weighted $\ell_{1}$ and the iteratively rotated $\ell_{1}$ method are very close in this case. In the Legendre polynomial expansion, the incorporation of iterative rotation improves the performance of the other methods. In the Chebyshev polynomial expansion, the improvement is minimal. This is related to the compromise of the property of $\boldsymbol{\Psi}$. Figure 6 depicts a comparison of $\boldsymbol{c}$ and $\tilde{\boldsymbol{c}}$, which shows the improvement of the sparsity in the similar manner as in function with high compressibility in Section 4.2 . Here, we plot coefficients with absolute values larger than $10^{-8}$ for demonstration purposes because other entries are negligible in the comparison of sparsity. The right column of Figure 6 also quantitatively illustrates sparsity enhancement. 


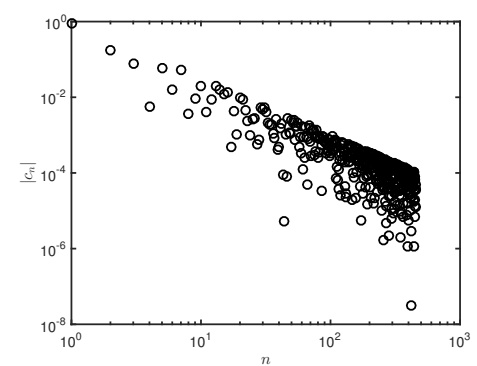

(a) Legendre $\left|c_{n}\right|$

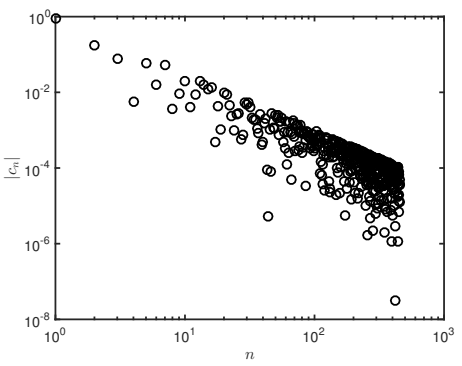

(d) Chebyshev $\left|c_{n}\right|$

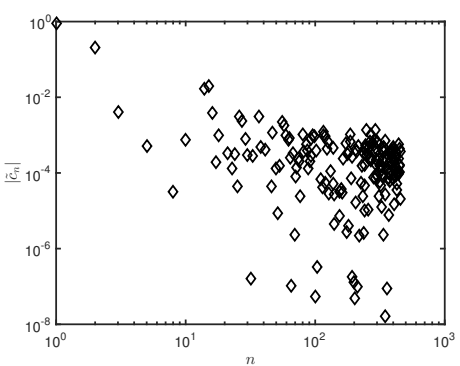

(b) Legendre $\left|\tilde{c}_{n}\right|$

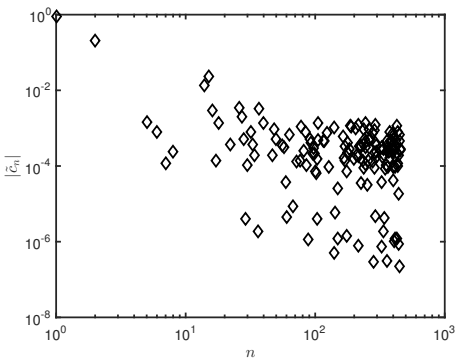

(e) Chebyshev $\left|\tilde{c}_{n}\right|$

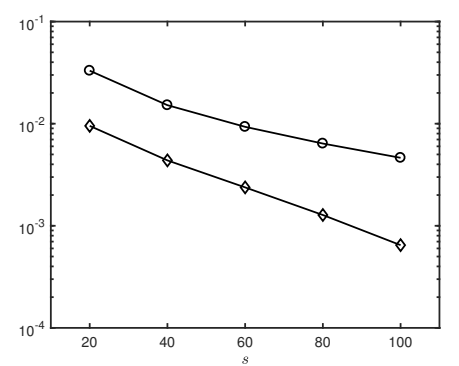

(c) Legendre comparison of sparsity

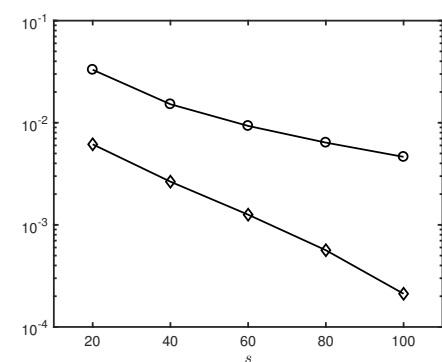

(f) Chebyshev comparison of sparsity

Figure 4: Results for the highly compressible function. Left column: absolute values of exact coefficients $c_{n}$; middle column: absolute values of coefficients $\tilde{c}_{n}$ after rotations using 160 samples; right column: comparison of $\frac{\left\|\boldsymbol{c}-\boldsymbol{c}_{s}\right\|_{1}}{\sqrt{s}}$ ("o") and $\frac{\left\|\tilde{\boldsymbol{c}}-\tilde{\boldsymbol{c}}_{s}\right\|_{1}}{\sqrt{s}}($ " $\diamond$ ") with different $s$.

\subsection{Korteweg-de Vries equation}

As an example application of our new method to a more complicated and nonlinear differential equation, we consider the Korteweg-de Vries (KdV) equation with time-dependent additive noise [32]:

$$
\begin{aligned}
& u_{t}(x, t ; \boldsymbol{\xi})-6 u(x, t ; \boldsymbol{\xi}) u_{x}(x, t ; \boldsymbol{\xi})+u_{x x x}(x, t ; \boldsymbol{\xi})=f(t ; \boldsymbol{\xi}), \quad x \in(-\infty, \infty), \\
& u(x, 0 ; \boldsymbol{\xi})=-2 \operatorname{sech}^{2}(x) .
\end{aligned}
$$

We model $f(t ; \boldsymbol{\xi})$ as a random field represented by the following KL expansion:

$$
f(t ; \boldsymbol{\xi})=\sigma \sum_{i=1}^{d} \sqrt{\lambda_{i}} \phi_{i}(t) \xi_{i}
$$

where $\sigma$ is a constant and $\left\{\lambda_{i}, \phi_{i}(t)\right\}_{i=1}^{d}$ are eigenpairs of the exponential covariance kernel as in Eqs. (43) and (44), respectively. In this problem, we set $l_{c}=0.25$ and $d=10$ $\left(\sum_{i=1}^{d} \lambda_{i}>0.96 \sum_{i=1}^{\infty} \lambda_{i}\right)$. In this case, the exact one-soliton solution is

$$
u(x, t ; \boldsymbol{\xi})=\sigma \sum_{i=1}^{d} \sqrt{\lambda_{i}} \xi_{i} \int_{0}^{t} \phi_{i}(y) \mathrm{d} y-2 \operatorname{sech}^{2}\left(x-4 t+6 \sigma \sum_{i=1}^{d} \sqrt{\lambda_{i}} \xi_{i} \int_{0}^{t} \int_{0}^{z} \phi_{i}(y) \mathrm{d} y \mathrm{~d} z\right) .
$$

The QoI is chosen to be $u(x, t ; \boldsymbol{\xi})$ at $x=6, t=1$ with $\sigma=0.4$. Because an analytical expression for $\phi_{i}$ is available, we can compute the integrals in Eq. 49. with high accuracy. Denoting

$$
A_{i}=\sqrt{\lambda_{i}} \int_{0}^{1} \phi_{i}(y) \mathrm{d} y, \quad B_{i}=\sqrt{\lambda_{i}} \int_{0}^{1} \int_{0}^{z} \phi_{i}(y) \mathrm{d} y \mathrm{~d} z, \quad i=1,2, \cdots, d,
$$



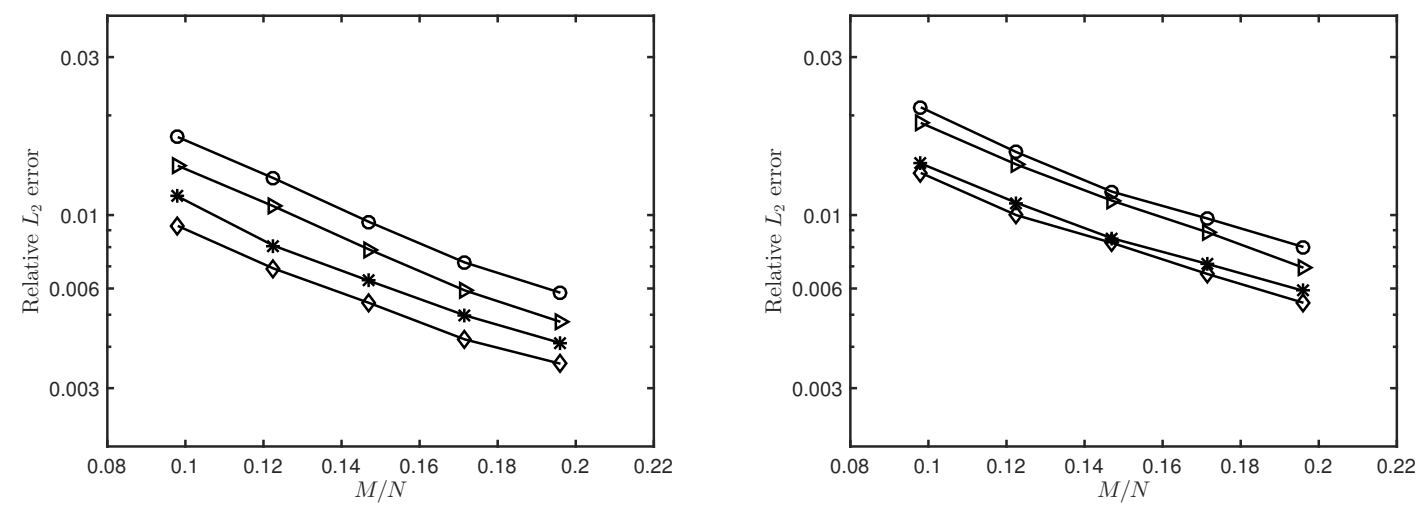

Figure 5: Results for the elliptic equation. Left: Legendre polynomial expansion (when $\xi_{i}$ are i.i.d. uniform random variables). Right: Chebyshev polynomial expansion (when $\xi_{i}$ are i.i.d.

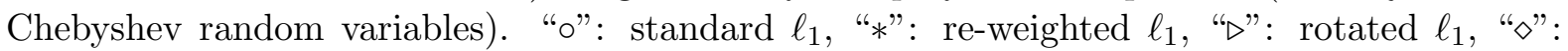
re-weighted+rotated $\ell_{1}$.

the analytical solution is

$$
\left.u(x, t ; \boldsymbol{\xi})\right|_{x=6, t=1}=\sigma \sum_{i=1}^{d} A_{i} \xi_{i}-2 \operatorname{sech}^{2}\left(2+6 \sigma \sum_{i=1}^{d} B_{i} \xi_{i}\right) .
$$

We use a fourth-order gPC expansion to approximate the solution, i.e., $P=4$, and the number of $\mathrm{gPC}$ basis functions $N=1001$. The $L_{2}$ error of the Legendre and Chebyshev polynomial expansions are presented in Figure 7. In this example, the combined iterative rotation and re-weighted $\ell_{1}$ method outperforms all other approaches. However, in the Chebyshev polynomial expansion, when the sample size is small (i.e., $M / N<0.12$ ) and if we only use the rotational method, the result is not as good as that determined by the standard $\ell_{1}$ minimization. This phenomenon also is related to the compromise in the property of $\boldsymbol{\Psi}$. Figure 8 presents a comparison of $\boldsymbol{c}$ and $\tilde{\boldsymbol{c}}$ (obtained using 180 samples), showing the sparsity improvement via the iterative rotation method. Coefficients with absolute values smaller than $10^{-8}$ are not presented as they are negligible in the comparison of sparsity. In addition, Figure 8 demonstrates the sparsity enhancement of the sparsity quantitatively.

\subsection{High-dimensional function}

In this example, we illustrate the potential capability of the rotational method for dealing with higher-dimensional problems. Specifically, we select a function similar to the first example (Section 4.1) but with much higher dimensionality:

$$
u(\boldsymbol{\xi})=\sum_{i=1}^{d} \xi_{i}+0.25\left(\sum_{i=1}^{d} \xi_{i} / \sqrt{i}\right)^{2}, \quad d=100 .
$$

The total number of basis functions for this example is $N=5151$. The relative error is computed with a level-3 sparse grid method. Hence, the numerical integrals are exact. The results are presented in Figure 9 As before, our iterative rotation approach outperforms the existing $\ell_{1}$ methods. Figure 10 features a comparison of $\boldsymbol{c}$ and $\tilde{\boldsymbol{c}}$, showing the sparsity enhancement of the sparsity using the iterative rotation method. Coefficients $\tilde{c}_{n}$ (obtained with 1200 samples) with absolute values smaller than $10^{-4}$ are not presented as they are two magnitudes smaller than the dominating ones and are negligible in the comparison of sparsity. The sparsity enhancement is illustrated quantitatively on the right column of Figure 10. Notably, for general high-dimensional problems, simply truncating the gPC expansion up to a certain order is not efficient because the number of basis grows exponentially. Hence, a 


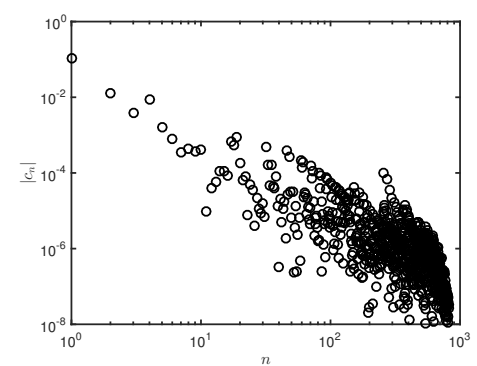

(a) Legendre $\left|c_{n}\right|$

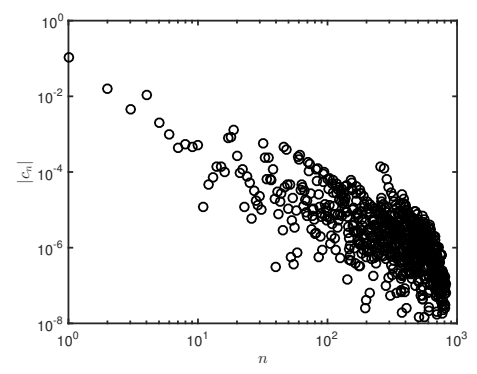

(d) Chebyshev $\left|c_{n}\right|$

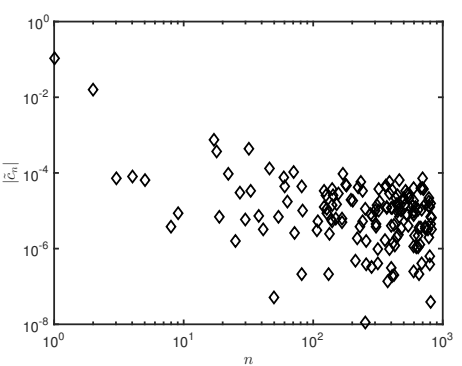

(b) Legendre $\left|\tilde{c}_{n}\right|$

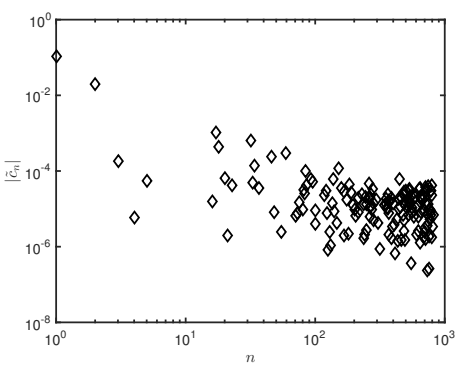

(e) Chebyshev $\left|\tilde{c}_{n}\right|$

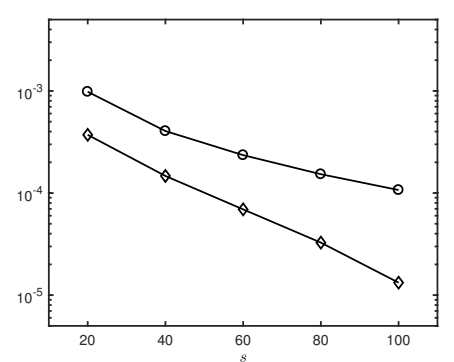

(c) Legendre comparison of sparsity

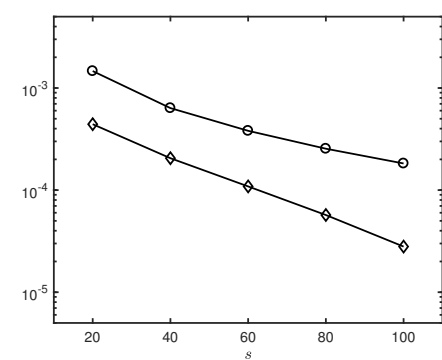

(f) Chebyshev comparison of sparsity

Figure 6: Results for the elliptic equation. Left column: absolute value of exact coefficients $\left|c_{n}\right|$; middle column: absolute values of coefficients $\tilde{c}_{n}$ after rotations using 160 samples; right column: comparison of $\frac{\left\|\boldsymbol{c}-\boldsymbol{c}_{s}\right\|_{1}}{\sqrt{s}}\left(\right.$ "०") and $\frac{\left\|\tilde{\boldsymbol{c}}-\tilde{\boldsymbol{c}}_{s}\right\|_{1}}{\sqrt{s}}(" \diamond$ ") with different $s$.

good approach for high-dimensional problems is to integrate our iterative rotation method with a method to reduce $d$ (e.g., ANOVA [54, SIR [31]) or to reduce $N$ (e.g., adaptive basis selection [25]).

\subsection{Increase in the mutual coherence}

As we point out in Section 3.4 , the property of $\Psi^{(l)}$ becomes less favorable for $\ell_{1}$ minimization as we use Legendre and Chebyshev polynomials in the expansion. Here, we use the mutual coherence $\mu(\boldsymbol{\Psi})$ (see Eq. (36)) to demonstrate this phenomenon. We employ the ridge function setup in example $1(d=12, P=3, N=455)$, and we use the exact rotation matrix $\mathbf{A}$ in Eq. (39) to illustrate how the mutual coherence changes. The matrix $\boldsymbol{\Psi}$ after rotation is computed as $\Psi_{i j}=\psi_{j}\left(\mathbf{A} \boldsymbol{\xi}^{i}\right)$, where $\mathbf{A}$ is given in Eq. (39). We repeat the computing of $\mu(\boldsymbol{\Psi})$ with 50 independent sets of $\left\{\boldsymbol{\xi}^{i}\right\}_{i=1}^{180}$ and present the average value in Table 1. As we expected, for the Hermite polynomial, $\mu$ does not change, while, for other types of polynomials, it increases. Further, the increase in the Chebyshev polynomial is larger than that of the Legendre polynomials. This provides a partial explanation as to why the rotational method for Chebyshev polynomial is less efficient than for the Legendre polynomial in some of our test cases. Although theoretical analysis is not available at this time, Table 1 provides an intuitive understanding of the algorithm.

\section{Conclusions}

In this work, we extend our previous work on rotation-based [30] and iterative-rotation algorithm [56] of Hermite polynomial expansion by providing a general framework for enhancing sparsity of gPC expansion by using an alternating direction method to identify a 

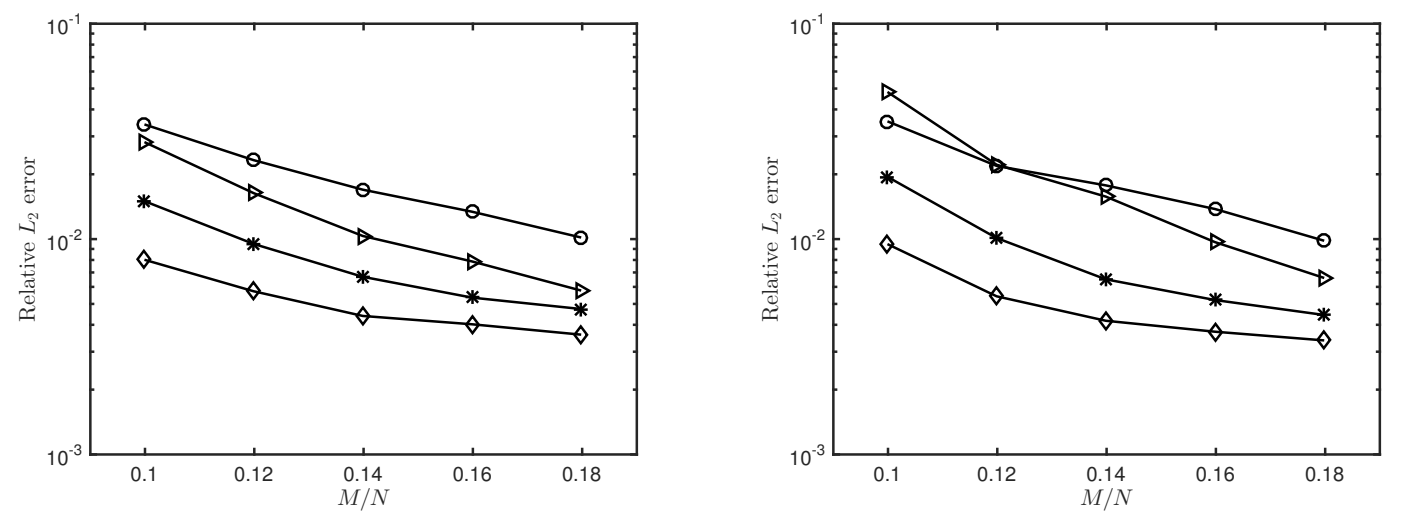

Figure 7: Results for the Korteweg-de Vries equation. Left: Legendre polynomial expansion (when $\xi_{i}$ are i.i.d. uniform random variables). Right: Chebyshev polynomial expansion (when $\xi_{i}$ are i.i.d. Chebyshev random variables). "o": standard $\ell_{1}$, "*": re-weighted $\ell_{1}$, "ฉ": rotated $\ell_{1}$, "»": re-weighted+rotated $\ell_{1}$.

Table 1: Comparison of mutual coherence before and after rotation for different types of polynomial expansions. $d=12, P=3, N=455, M=180$.

\begin{tabular}{c|ccc}
\hline \hline & Legendre & Chebyshev & Hermite \\
\hline$\mu(\boldsymbol{\Psi})$ before rotation & 0.15 & 0.15 & 0.40 \\
$\mu(\boldsymbol{\Psi})$ after rotation & 0.45 & 0.50 & 0.40 \\
\hline \hline
\end{tabular}

rotation iteratively. As such, it improves the accuracy of the compressive sensing method to construct the gPC expansions from a small amount of data. The rotation is decided by seeking the directions of maximum variation for the QoI through SVD of the gradients at different points in the parameter space. We also demonstrate that our previously developed iterative method for Hermite polynomial expansion [56] is a special case of this general framework.

We combine the iterative rotations with $\ell_{1}$ minimization. Iterative rotations also can be integrated with other optimization methods to solve the compressive sensing problem, e.g., orthogonal matching pursuit (OMP) [6], $\ell_{1-2}$ minimization [59], and transformed $\ell_{1}$ [21]. Alternatively, it is possible to alleviate this problem by using the Gram-Schmidt method to reorthogonalize the measurement matrix [15] or using a better sampling and preconditioning scheme [26]. In addition, it is possible to further improve the accuracy and reduce the number of samples by integrating our method with advanced sampling strategies (e.g., 22]), adaptive basis selection method (e.g., [25]), Bayesian compressive sensing method (e.g., [28]), a better initial guess for the iteration (e.g.,[57), etc. This is specifically useful for problems where the experiments or simulations are costly. The resulting surrogate model $u_{g}$ can be used to study parameter sensitivities, and it can be useful in inverse problems based on the Bayesian framework.

Along with our previous work, we have demonstrated the effectiveness of the rotational method for the Hermite and Legendre polynomial expansions. These two are the most useful gPC expansions used in the UQ studies as the Gaussian and uniform random variables are the most widely used in practice. Our method requires fewer samples of QoI to construct surrogate models, which can be a great savings of experimental or computational resources. As such, it is useful for most UQ problems. Of note, the main limitation of applying our method to other types of gPC expansions is the possible degeneration of the property of the measurement matrix. For example, for the Laguerre polynomial expansion, numerical tests (not presented in this work) show that our method does not work for some cases-even if the UQ representation has low dimensional structure. A systematic numerical analysis on 


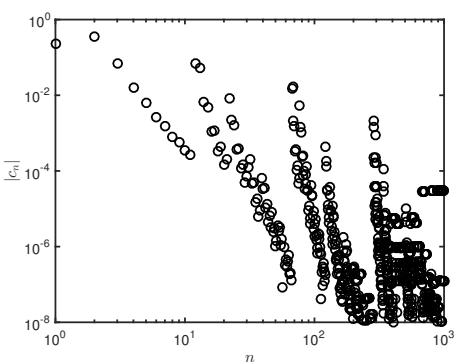

(a) Legendre $\left|c_{n}\right|$

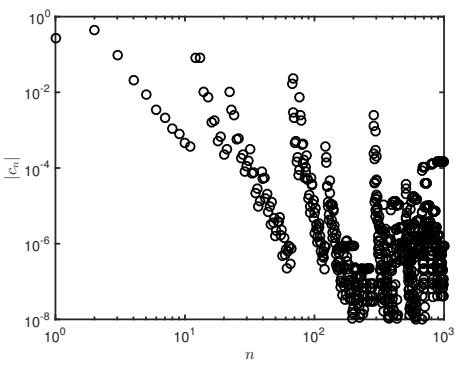

(d) Chebyshev $\left|c_{n}\right|$



(b) Legendre $\left|\tilde{c}_{n}\right|$

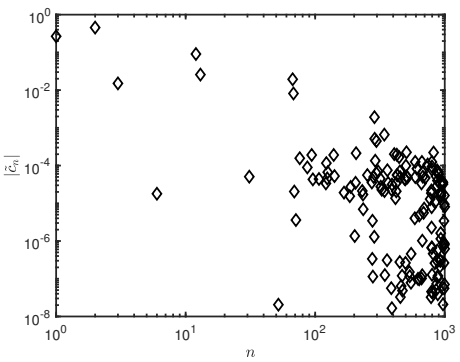

(e) Chebyshev $\left|\tilde{c}_{n}\right|$



(c) Legendre comparison of sparsity

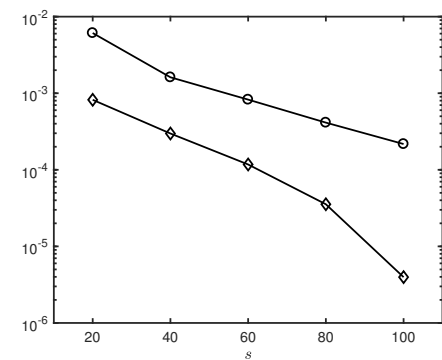

(f) Chebyshev comparison of sparsity

Figure 8: Results for the KdV equation. Left column: absolute values of exact coefficients $c_{n}$; middle column: absolute values coefficients $\tilde{c}_{n}$ after rotations using 180 samples; right column: comparison of $\frac{\left\|\boldsymbol{c}-\boldsymbol{c}_{s}\right\|_{1}}{\sqrt{s}}$ ("○") and $\frac{\left\|\tilde{\boldsymbol{c}}-\tilde{\boldsymbol{c}}_{s}\right\|_{1}}{\sqrt{s}}$ ("ऽ") with different $s$.

the convergence will be the focus of our future work. At this time, an intuitive guidance is that our method works well when the PDF is symmetric (or approximately symmetric) with respect to the origin under rotation.

Finally, as we point out in Section 3.2 , removing the constraint $\mathbf{A} \mathbf{A}^{\top}=\mathbf{I}$ may result in a more accurate $u_{g}$. Also, a nonlinear map from $\boldsymbol{\xi}$ to $\boldsymbol{\eta}$ may work even better. These improvements allow us to explore low-dimensional structures of the system more comprehensively, and will be addressed in our future work.

\section{Acknowledgments}

We would like to thank Dr. Nathan Baker for fruitful discussions. The research described in this paper was conducted under the Laboratory Directed Research and Development Program at Pacific Northwest National Laboratory, a multiprogram national laboratory operated by Battelle for the U.S. Department of Energy. PNNL is operated by Battelle for the DOE under Contract DE-AC05-76RL01830. A portion of this work was supported by the of Energy, Office of Science, Office of Advanced Scientific Computing Research as part of the Collaboratory on Mathematics for Mesoscopic Modeling of Materials (CM4).

\section{References}

[1] Ben Adcock. Infinite-dimensional compressed sensing and function interpolation. Found. of Comput. Math., pages 1-41, 2017. 

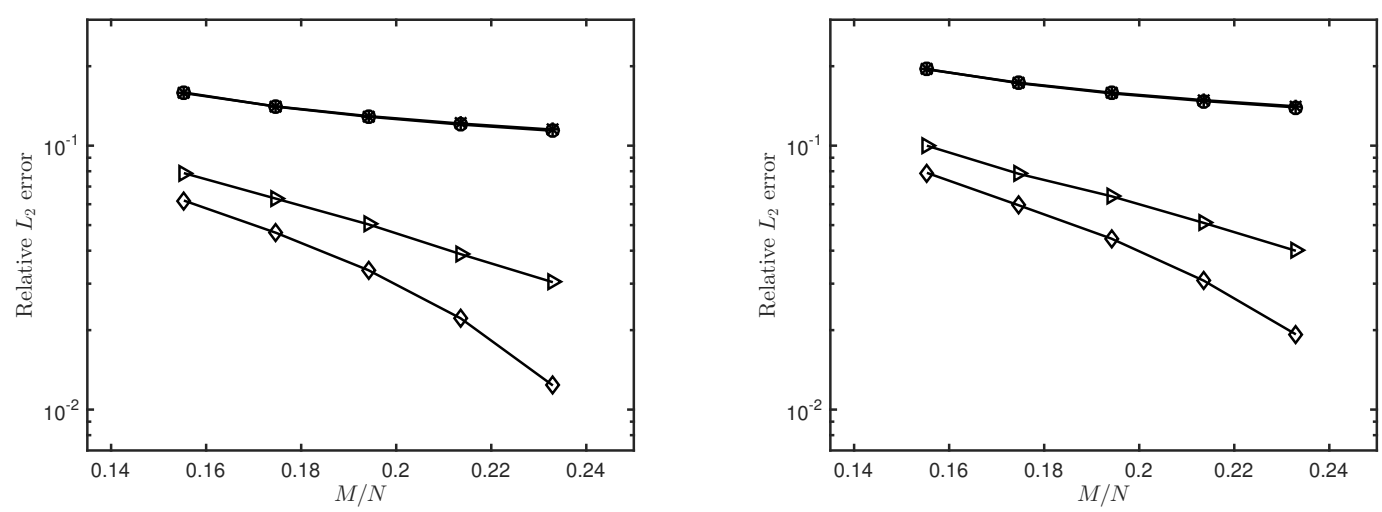

Figure 9: Results for the high-dimensional function. Left: Legendre polynomial expansion (when $\xi_{i}$ are i.i.d. uniform random variables). Right: Chebyshev polynomial expansion (when $\xi_{i}$ are i.i.d. Chebyshev random variables). "o": standard $\ell_{1}$, "*": re-weighted $\ell_{1}$, "ฉ": rotated $\ell_{1}$, "»": re-weighted+rotated $\ell_{1}$.

[2] Negin Alemazkoor and Hadi Meidani. Divide and conquer: An incremental sparsity promoting compressive sampling approach for polynomial chaos expansions. Comput. Methods Appl. Mech. Eng., 318:937-956, 2017.

[3] Negin Alemazkoor and Hadi Meidani. Divide and conquer: An incremental sparsity promoting compressive sampling approach for polynomial chaos expansions. Comput. Methods Appl. Mech. Engrg., 318:937-956, 2017.

[4] Ivo Babuška, Fabio Nobile, and Raul Tempone. A stochastic collocation method for elliptic partial differential equations with random input data. SIAM Rev., 52(2):317$355,2010$.

[5] Géraud Blatman and Bruno Sudret. Adaptive sparse polynomial chaos expansion based on least angle regression. J. Comput. Phys., 230(6):2345-2367, 2011.

[6] Alfred M. Bruckstein, David L. Donoho, and Michael Elad. From sparse solutions of systems of equations to sparse modeling of signals and images. SIAM Rev., 51(1):34-81, 2009.

[7] Robert H Cameron and William T Martin. The orthogonal development of non-linear functionals in series of fourier-hermite functionals. Ann. Math., pages 385-392, 1947.

[8] Emmanuel J. Candès. The restricted isometry property and its implications for compressed sensing. C. R. Math. Acad. Sci. Paris, 346(9-10):589-592, 2008.

[9] Emmanuel J Candès, Justin K Romberg, and Terence Tao. Stable signal recovery from incomplete and inaccurate measurements. Comm. Pure Applied Math., 59(8):12071223, 2006.

[10] Emmanuel J. Candès and Terence Tao. Decoding by linear programming. IEEE Trans. Inform. Theory, 51(12):4203-4215, 2005.

[11] Emmanuel J. Candès, Michael B. Wakin, and Stephen P. Boyd. Enhancing sparsity by reweighted $l_{1}$ minimization. J. Fourier Anal. Appl., 14(5-6):877-905, 2008.

[12] Paul G Constantine, Eric Dow, and Qiqi Wang. Active subspace methods in theory and practice: Applications to kriging surfaces. SIAM J. Sci. Comput., 36(4):A1500-A1524, 2014.

[13] Wei Dai and Olgica Milenkovic. Subspace pursuit for compressive sensing: Closing the gap between performance and complexity. Technical report, ILLINOIS UNIV AT URBANA-CHAMAPAIGN, 2008.

[14] Chandler Davis and William Morton Kahan. The rotation of eigenvectors by a perturbation. iii. SIAM J. Numer. Anal., 7(1):1-46, 1970. 


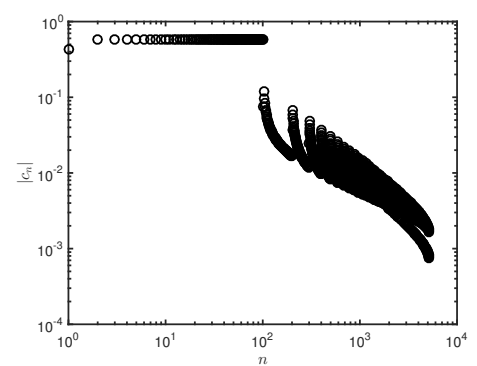

(a) Legendre $\left|c_{n}\right|$

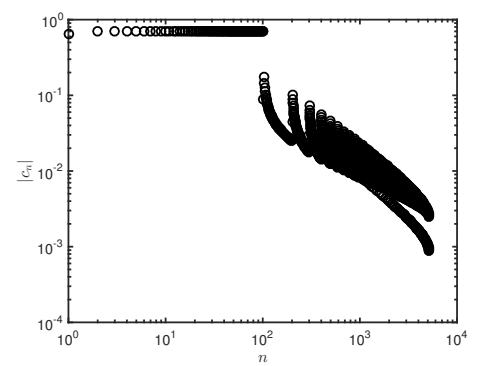

(d) Chebyshev $\left|c_{n}\right|$

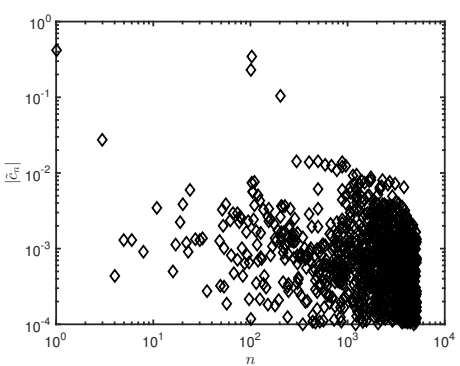

(b) Legendre $\left|\tilde{c}_{n}\right|$

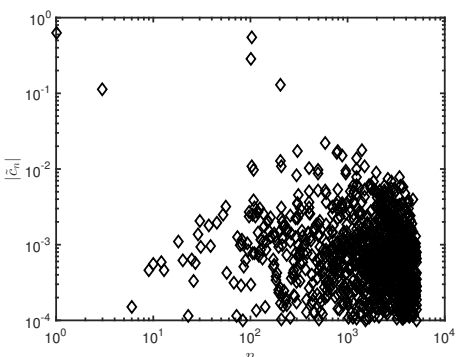

(e) Chebyshev $\left|\tilde{c}_{n}\right|$

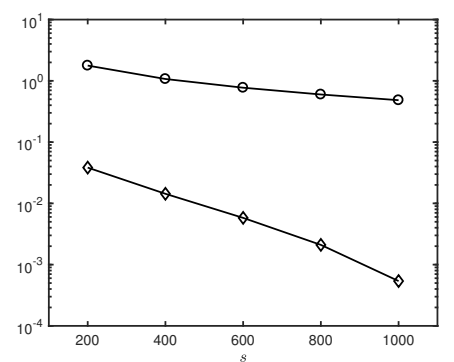

(c) Legendre comparison of sparsity

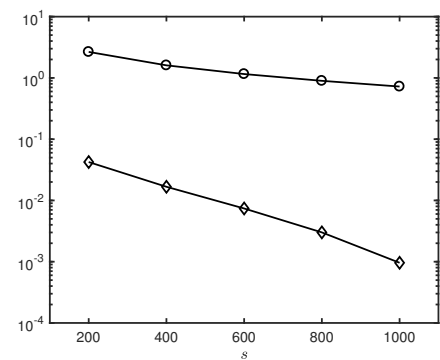

(f) Chebyshev comparison of sparsity

Figure 10: Results for the high-dimensional function. Left column: absolute values of exact coefficients $c_{n}$; middle column: absolute values of coefficients $\tilde{c}_{n}$ after rotations using 1200 samples; right column: comparison of $\frac{\left\|\boldsymbol{c}-\boldsymbol{c}_{s}\right\|_{1}}{\sqrt{s}}$ ("o") and $\frac{\left\|\tilde{\boldsymbol{c}}-\tilde{\boldsymbol{c}}_{s}\right\|_{1}}{\sqrt{s}}($ " $\diamond$ ") with different $s$.

[15] S Dey, T Mukhopadhyay, H Haddad Khodaparast, and S Adhikari. Fuzzy uncertainty propagation in composites using gram-schmidt polynomial chaos expansion. Appl. Math. Model., 40(7-8):4412-4428, 2016.

[16] David L Donoho. Compressed sensing. IEEE Trans. Inf. Theory, 52(4):1289-1306, 2006.

[17] David L. Donoho, Michael Elad, and Vladimir N. Temlyakov. Stable recovery of sparse overcomplete representations in the presence of noise. IEEE Trans. Inform. Theory, $52(1): 6-18,2006$.

[18] Alireza Doostan and Houman Owhadi. A non-adapted sparse approximation of PDEs with stochastic inputs. J. Comput. Phys., 230(8):3015-3034, 2011.

[19] Oliver G Ernst, Antje Mugler, Hans-Jörg Starkloff, and Elisabeth Ullmann. On the convergence of generalized polynomial chaos expansions. ESAIM: Math. Model. Numer. Anal., 46(2):317-339, 2012.

[20] Roger G. Ghanem and Pol D. Spanos. Stochastic finite elements: a spectral approach. Springer-Verlag, New York, 1991.

[21] Ling Guo, Jing Li, and Yongle Liu. Stochastic collocation methods via minimization of transformed l_1 penalty. arXiv preprint arXiv:1805.05416, 2018.

[22] Jerrad Hampton and Alireza Doostan. Compressive sampling of polynomial chaos expansions: Convergence analysis and sampling strategies. J. Comput. Phys., 280(0):363$386,2015$.

[23] Jerrad Hampton and Alireza Doostan. Basis adaptive sample efficient polynomial chaos (base-pc). J. Comput. Phys., 371:20-49, 2018. 
[24] Alan J Hoffman and Helmut W Wielandt. The variation of the spectrum of a normal matrix. In Selected Papers Of Alan J Hoffman: With Commentary, pages 118-120. World Scientific, 2003.

[25] John D Jakeman, Michael S Eldred, and Khachik Sargsyan. Enhancing $\ell_{1}$-minimization estimates of polynomial chaos expansions using basis selection. J. Comput. Phys., 289:18-34, 2015.

[26] John D Jakeman, Akil Narayan, and Tao Zhou. A generalized sampling and preconditioning scheme for sparse approximation of polynomial chaos expansions. SIAM J. Sci. Comput., 39(3):A1114-A1144, 2017.

[27] M Jardak, Chau-Hsing Su, and George Em Karniadakis. Spectral polynomial chaos solutions of the stochastic advection equation. J. Sci. Comput., 17(1-4):319-338, 2002.

[28] Georgios Karagiannis, Bledar A Konomi, and Guang Lin. A Bayesian mixed shrinkage prior procedure for spatial-stochastic basis selection and evaluation of $\mathrm{gPC}$ expansions: Applications to elliptic SPDEs. J. Comput. Phys., 284:528-546, 2015.

[29] Huan Lei, Xiu Yang, Zhen Li, and George Em Karniadakis. Systematic parameter inference in stochastic mesoscopic modeling. J. Comput. Phys., 330:571-593, 2017.

[30] Huan Lei, Xiu Yang, Bin Zheng, Guang Lin, and Nathan A Baker. Constructing surrogate models of complex systems with enhanced sparsity: quantifying the influence of conformational uncertainty in biomolecular solvation. SIAM Multiscale Model. Simul., 13(4):1327-1353, 2015.

[31] Ker-Chau Li. Sliced inverse regression for dimension reduction. J. Am. Stat. Assoc., 86(414):316-327, 1991.

[32] Guang Lin, Leopold Grinberg, and George Em Karniadakis. Numerical studies of the stochastic korteweg-de vries equation. J. Comput. Phys., 213(2):676-703, 2006.

[33] Xiang Ma and Nicholas Zabaras. An adaptive high-dimensional stochastic model representation technique for the solution of stochastic partial differential equations. $J$. Comput. Phys., 229(10):3884-3915, 2010.

[34] D. Needell. Noisy signal recovery via iterative reweighted $l_{1}$ minimization. In Proc. Asilomar Conf. on Signal Systems and Computers, Pacific Grove, CA, 2009.

[35] Hisanao Ogura. Orthogonal functionals of the Poisson process. IEEE Trans. Inf. Theory, 18(4):473-481, 1972.

[36] Ji Peng, Jerrad Hampton, and Alireza Doostan. A weighted $\ell_{1}$-minimization approach for sparse polynomial chaos expansions. J. Comput. Phys., 267(0):92-111, 2014.

[37] Ji Peng, Jerrad Hampton, and Alireza Doostan. On polynomial chaos expansion via gradient-enhanced $\ell_{1}$-minimization. J. Comput. Phys., 310:440-458, 2016.

[38] Holger Rauhut and Rachel Ward. Sparse legendre expansions via $l_{1}$-minimization. $J$. Approx. Theory, 164(5):517-533, May 2012.

[39] Holger Rauhut and Rachel Ward. Interpolation via weighted $\ell_{1}$ minimization. Appl. Comput. Harmon. Anal., 40(2):321 - 351, 2016.

[40] Trent Michael Russi. Uncertainty quantification with experimental data and complex system models. PhD thesis, UC Berkeley, 2010.

[41] Khachik Sargsyan, Cosmin Safta, Habib N Najm, Bert J Debusschere, Daniel Ricciuto, and Peter Thornton. Dimensionality reduction for complex models via bayesian compressive sensing. Int. J. Uncertain. Quan., 4(1), 2014.

[42] S. Smolyak. Quadrature and interpolation formulas for tensor products of certain classes of functions. Sov. Math. Dokl., 4:240-243, 1963.

[43] Gilbert W Stewart. Matrix perturbation theory. 1990.

[44] Menner A Tatang, Wenwei Pan, Ronald G Prinn, and Gregory J McRae. An efficient method for parametric uncertainty analysis of numerical geophysical models. $J$. Geophys. Res-Atmos. (1984-2012), 102(D18):21925-21932, 1997. 
[45] Ramakrishna Tipireddy and Roger Ghanem. Basis adaptation in homogeneous chaos spaces. J. Comput. Phys., 259(0):304-317, 2014.

[46] Panagiotis Tsilifis, Xun Huan, Cosmin Safta, Khachik Sargsyan, Guilhem Lacaze, Joseph C Oefelein, Habib N Najm, and Roger G Ghanem. Compressive sensing adaptation for polynomial chaos expansions. arXiv preprint arXiv:1801.01961, 2018.

[47] E. van den Berg and M. P. Friedlander. SPGL1: A solver for large-scale sparse reconstruction, June 2007. http://www.cs.ubc.ca/labs/scl/spgl1.

[48] E. van den Berg and M. P. Friedlander. Probing the Pareto frontier for basis pursuit solutions. SIAM J. Sci. Comput., 31(2):890-912, 2008.

[49] Dongbin Xiu and Jan S. Hesthaven. High-order collocation methods for differential equations with random inputs. SIAM J. Sci. Comput., 27(3):1118-1139, 2005.

[50] Dongbin Xiu and George Em Karniadakis. The Wiener-Askey polynomial chaos for stochastic differential equations. SIAM J. Sci. Comput., 24(2):619-644, 2002.

[51] Zhiqiang $\mathrm{Xu}$ and Tao Zhou. On sparse interpolation and the design of deterministic interpolation points. SIAM J. Sci. Comput., 36(4):A1752-A1769, 2014.

[52] Liang Yan, Ling Guo, and Dongbin Xiu. Stochastic collocation algorithms using $l_{1^{-}}$ minimization. Int. J. Uncertain. Quant., 2(3):279-293, 2012.

[53] Xiu Yang, David A Barajas-Solano, W Steven Rosenthal, and Alexandre M Tartakovsky. PDF estimation for power grid systems via sparse regression. arXiv preprint arXiv:1708.08378, 2017.

[54] Xiu Yang, Minseok Choi, Guang Lin, and George Em Karniadakis. Adaptive ANOVA decomposition of stochastic incompressible and compressible flows. J. Comput. Phys., 231(4):1587-1614, 2012.

[55] Xiu Yang and George Em Karniadakis. Reweighted $\ell_{1}$ minimization method for stochastic elliptic differential equations. J. Comput. Phys., 248(1):87-108, 2013.

[56] Xiu Yang, Huan Lei, Nathan Baker, and Guang Lin. Enhancing sparsity of hermite polynomial expansions by iterative rotations. J. Comput. Phys., 307:94-109, 2016.

[57] Xiu Yang, Weixuan Li, and Alexandre Tartakovsky. Sliced-inverse-regression-aided rotated compressive sensing method for uncertainty quantification. arXiv preprint arXiv:1709.07937, 2017.

[58] Xiu Yang, Xiaoliang Wan, and George Em Karniadakis. Generalized polynomial chaos: approximation through change of measure. 4th Intern. Cong. Comput. Engrg. Sci., 2013.

[59] Penghang Yin, Yifei Lou, Qi He, and Jack Xin. Minimization of $\ell_{1-2}$ for compressed sensing. SIAM J. Sci. Comput., 37(1):A536-A563, 2015.

[60] Zheng Zhang, Xiu Yang, Ivan V Oseledets, George E Karniadakis, and Luca Daniel. Enabling high-dimensional hierarchical uncertainty quantification by ANOVA and tensortrain decomposition. IEEE Trans. Comput.-Aided Des. Integr. Circuits and Syst., 34(1):63-76, 2015. 